\title{
Restriction associated DNA-genotyping at multiple spatial scales in Arabidopsis lyrata reveals signatures of pathogen-mediated selection
}

\author{
James Buckley ${ }^{1,2^{*}}$, Eric B. Holub ${ }^{3}$, Marcus A. Koch ${ }^{4}$, Philippine Vergeer ${ }^{5}$ and Barbara K. Mable ${ }^{1}$
}

\begin{abstract}
Background: Genome scans based on outlier analyses have revolutionized detection of genes involved in adaptive processes, but reports of some forms of selection, such as balancing selection, are still limited. It is unclear whether high throughput genotyping approaches for identification of single nucleotide polymorphisms have sufficient power to detect modes of selection expected to result in reduced genetic differentiation among populations. In this study, we used Arabidopsis lyrata to investigate whether signatures of balancing selection can be detected based on genomic smoothing of Restriction Associated DNA sequencing (RAD-seq) data. We compared how different sampling approaches (both within and between subspecies) and different background levels of polymorphism (inbreeding or outcrossing populations) affected the ability to detect genomic regions showing key signatures of balancing selection, specifically elevated polymorphism, reduced differentiation and shifts towards intermediate allele frequencies. We then tested whether candidate genes associated with disease resistance (R-gene analogs) were detected more frequently in these regions compared to other regions of the genome.

Results: We found that genomic regions showing elevated polymorphism contained a significantly higher density of $R$-gene analogs predicted to be under pathogen-mediated selection than regions of non-elevated polymorphism, and that many of these also showed evidence for an intermediate site-frequency spectrum based on Tajima's D. However, we found few genomic regions that showed both elevated polymorphism and reduced $\mathrm{F}_{\text {ST }}$ among populations, despite strong background levels of genetic differentiation among populations. This suggests either insufficient power to detect the reduced population structure predicted for genes under balancing selection using sparsely distributed RAD markers, or that other forms of diversifying selection are more common for the R-gene analogs tested.
\end{abstract}

Conclusions: Genome scans based on a small number of individuals sampled from a wide range of populations were sufficient to confirm the relative scarcity of signatures of balancing selection across the genome, but also identified new potential disease resistance candidates within genomic regions showing signatures of balancing selection that would be strong candidates for further sequencing efforts.

Keywords: Balancing selection, Arabidopsis lyrata, Genome scan, RAD-seq, Polymorphism, Mating system, Pathogens, R-genes, Disease resistance

\footnotetext{
* Correspondence: james.buckley@env.ethz.ch

'Institute of Biodiversity, Animal Health and Comparative Medicine, College

of Medical, Veterinary and Life Sciences, University of Glasgow, Glasgow G12 $8 \mathrm{QQ}$, UK

${ }^{2}$ Adaptation to a Changing Environment, Institute of Integrative Biology, ETH

Zürich, CH-8092 Zürich, Switzerland

Full list of author information is available at the end of the article
}

(c) The Author(s). 2018 Open Access This article is distributed under the terms of the Creative Commons Attribution 4.0 International License (http://creativecommons.org/licenses/by/4.0/), which permits unrestricted use, distribution, and reproduction in any medium, provided you give appropriate credit to the original author(s) and the source, provide a link to the Creative Commons license, and indicate if changes were made. The Creative Commons Public Domain Dedication waiver (http://creativecommons.org/publicdomain/zero/1.0/) applies to the data made available in this article, unless otherwise stated. 


\section{Background}

High throughput sequencing and associated genotyping methods have revolutionized population genomic studies of population structure and selection and been applied to a wide range of species [1]. Such approaches have proved particularly useful for resolving phylogeographic structure in non-model taxa [2-4], but also for detecting signatures of selection across the genome and therefore identifying novel candidate loci being influenced by selection [1, 4-9]. Nevertheless, these different aims often greatly differ in their strategy for sampling populations and individuals of a species, with phylogeographic studies often sampling many populations to maximise range-wide variation and studies aimed at detecting signatures of selection sampling more extensively within a smaller number of populations. There is also a bias towards detecting signatures of divergent, positive selection, rather than other forms of selection [5, 10, 11], where extensive within population sampling is thought to be critical [12].

Evidence for balancing selection is thus more limited than directional selection and mostly restricted to studies involving whole genome or gene sequencing in a small set of organisms, including humans and pathogenic organisms [13-15], and more recently species of Arabidopsis [16] and Drosophila [17]. An important question is therefore whether this scarcity of evidence is due to balancing selection being limited in its role in shaping genome-wide patterns of diversity (e.g. [13]), or due to the challenges of identifying signatures of balancing selection as opposed to other types of selection [10].

Balancing selection maintains adaptive genetic variation among and within populations through different mechanisms: frequency-dependent selection, heterozygote advantage and fluctuating selection in time and space [18-20]. Spatial variation in selection can result in variation being reduced locally, but maintained at a broader geographic scale in different geographic regions or environments [21]. Balancing selection is generally characterized by an increased coalescence time compared to neutral expectations, leading to an excess of polymorphism in an extended genomic region linked to the selected variant, an excess of intermediate frequency alleles, increased heterozygosity, and reduced differentiation among populations $[22,23]$. Several of these signatures of balancing selection, in particular elevated polymorphism or reduced differentiation, could be easier to detect by sampling few individuals from many diverged populations, rather than extensively within a few populations (e.g. [24]).

Testing for increased polymorphism or extended linkage disequilibrium (LD) is a useful starting point for detecting balancing selection, but such patterns alone are potentially confounded by similar signatures expected under diversifying selection or selective sweeps, respectively, as well as different rates of recombination across the genome [22]. Combining these signatures with additional population genetic statistics, such as reduced genetic differentiation $\left(\mathrm{F}_{\mathrm{ST}}\right)$ relative to neutral expectations or shifts in the site frequency spectra towards intermediate frequency alleles (positive Tajima's D values [25]), can provide more robust support for balancing selection $[4,7,26]$. However, many of these additional signatures of balancing selection can also reflect non-selective processes. For example, elevated Tajima's D values can be produced by a demographic bottleneck [27] and high background levels of polymorphism can make it more difficult to detect an excess of genetic diversity or heterozgyosity at a locus under balancing selection, particularly in highly outcrossing populations, where variation in rates of recombination across the genome might also be high [22]. It is therefore important to be able to account for demographic history when interpreting patterns of selection.

Most studies so far have used whole gene sequences or whole genome re-sequencing to detect genomic signatures of balancing selection, but genotyping by sequencing data such as Restriction Associated DNA (RAD) sequencing could also be used to detect such signatures [28]. The short length of RAD markers (around $100 \mathrm{bp}$ ) and uneven spacing across genomes limits the use of traditional LD-based approaches (e.g. [29]), and the relatively low density of markers may limit the ability to detect SNPs linked to candidate genes under selection ([30], but see [9]). However, when reference genomes are available and higher numbers of loci used, sliding window-based approaches can be used to identify outlying genomic regions (groups of neighbouring loci) showing unusually high or low levels of polymorphism, genetic differentiation and allele frequencies within populations (e.g. [4, 7]). Identifying genomic regions showing multiple signatures of balancing selection provides a general test for how common this form of balancing selection is across the genome, but would also highlight candidate loci in these regions likely to be influenced by balancing selection.

The potential complexity in patterns of polymorphism at loci under balancing selection means that special attention should also be given to filtering and assembly of high throughput sequencing data. As many genes under this type of selection are members of large gene families characterized by copy number variation (e.g. [10, 31, 32]), filtering SNPs to minimise the amount of missing data or remove potential paralogs could inadvertently exclude the strongest candidates [33]. Furthermore, an excess of predicted polymorphism might result from alleles being incorrectly assembled or aligned to reference 
genomes. Thus, genome scans for balancing selection might also require both different levels of sampling and different filtering decisions than tests for other types of selection.

Genes involved in ligand-mediated recognition processes in animals and plants, such as immune genes, plant disease resistance genes ( $R$-genes), and genes controlling self-incompatibility are often cited as classic examples of balancing selection [20, 34]. In plants, large families of $R$-gene analogs (RGAs) contain particular motifs based on conserved structural features [35-37]. A leucine rich repeat (LRR) motif is a shared domain in the three main plant RGA families, including two classes of cell surface receptors (receptor-like kinases, RLKs; and receptor-like proteins, RLPs) and a class of cytoplasmic receptors (nucleotide binding site-leucine rich repeat proteins, NLRs). RGAs therefore make a good test panel for investigating the power of genome scan approaches to detect balancing selection.

Whole genome sequencing of 20 wild A. thaliana accessions showed that the density of NLR encoding genes positively predicted levels of polymorphism across the genome [38]. Interestingly, sequencing of RGAs from a broad-scale sampling of Arabidopsis thaliana accessions has revealed genes showing clear signatures of balancing selection, but also others showing patterns more consistent with neutrality or directional selection [39-41]. As well as high levels of polymorphism, some plant NLR genes show presence and absence of functional alleles that confer resistance to specific pathogen genotypes, and many show copy number variation across related species [42]. By contrast, other RGA families, such as RLKs or RLPs, are not predicted to show such elevated polymorphism, as they are involved in more conserved interactions with PAMPs and MAMPs (Pathogen- and Microbe-associated molecular patterns) [35].

Given that most of our knowledge of RGAs is restricted to the highly selfing $A$. thaliana, outcrossing Arabidopsis relatives offer a great opportunity to explore the effects of differing ecology on such loci associated with adaptation [16, 43, 44]. Arabidopsis lyrata has an annotated reference genome [45] and shows a restricted ecological distribution, with strong population structuring both within North America (A. lyrata subsp. lyrata, in the following simplified as A. l. lyrata) and Europe ( $A$. lyrata subsp. petraea, in the following simplified as $A$. $l$. petraea), as well as extensive differentiation between the subspecies $[5,46,47]$. A. l. petraea shows higher diversity than A. l. lyrata, with the highest diversity found in central European populations (Germany and Austria, thought to represent the centre of ancestral diversity in a glacial refugium; $[46,48,49]$, a region that also shows extensive introgression with related Arabidopsis taxa [50]. A recent study based on whole genome re-sequencing confirmed a bottleneck in North America, and postglacial expansion from Central Europe into Scandinavia and the United Kingdom [5], with relatively deep divergence times among the European regions. Thus, there is substantial and well-characterised population structure to provide a framework for investigating patterns of selection. In previous work, targeted sequencing of two $R$-gene analogs detected weak evidence for balancing selection in A. lyrata, despite strong evidence in A. thaliana, but emphasised that tests for selection are sensitive to the relative divergence among populations included in the analysis, since a stronger signature was detected by comparing subspecies rather than within subspecies [51].

North American populations also show variation in mating system within a limited geographic area in the Great Lakes regions [52-55]. Selfing populations show substantially reduced genetic diversity and heterozygosity compared to outcrossing populations in the same geographic region, as well as evidence of independent post-glacial colonization [54] and a severe bottleneck evidenced by a reduced number of self-incompatibility alleles [56]. The low levels of background polymorphism observed in these selfing populations could make it easier to detect elevated diversity and heterozygosity at loci under balancing selection.

In this study, we used RAD-sequencing of A. lyrata samples from the two subspecies to: (1) compare the resolution of broad- and finer-scale patterns of phylogeographic structure and genetic variation within these subspecies with previous studies using microsatellite loci or single nucleotide polymorphism genotyping based on relatively few genes; (2) identify regions of the genome showing deviations from this neutral structure characteristic of balancing selection; and (3) test the ability of different sampling strategies to detect balancing selection using a test panel of RGAs. We focused our analyses on different subsets of samples from the two subspecies to identify genome-wide patterns of polymorphism and divergence. Specifically, we tested for: (a) high diversity and heterozygosity in otherwise low diversity genetic backgrounds, using selfing populations of $A$. l. lyrata from the North American Great Lakes; and (b) reduced population genetic structure and changes in site frequency spectra, using different sets of outcrossing populations sharing a recent postglacial demographic history. We also tested the sensitivity of conclusions to filtering strategies, and compared genome scans using both individual RAD loci and smoothed genomic regions based on sliding window analyses.

We show that genotyping just a few individuals per location at multiple spatial scales can both resolve patterns of phylogeographic structure, but also identify genomic regions showing signatures of balancing or 
diversifying selection that contain candidate disease resistance loci.

\section{Results}

Spatially arranged genetic structure, diversity and heterozygosity across multiple spatial scales in A. lyrata We genotyped 91 individuals across the subspecies of $A$. lyrata using RAD-seq (Fig. 1a, b; Additional file 1: Table S1a,b) at 5942 loci present in all individuals and confirmed phylogeographic patterns consistent with neutral expectations, both in terms of levels of polymorphism in different geographic regions and genetic relationships among populations. Despite there being on average more reads for European samples (Additional file 1: Figure S1a), a similar number of reads aligned uniquely to the reference sequence (Additional file 1: Figure S1b), and to multiple places in the genome (Additional file 1: Figure S1c) for European and North American samples. However, as expected, given that the reference genome sequence derives from a North American individual (the IND population; [45]), there were significantly lower numbers of unaligned reads for North American than European samples (Additional file 1: Figure S1d). Levels of polymorphism were lowest in North American selfing samples and highest in central European samples (Fig. 1d; Additional file 1: Table S2). Comparisons between observed $\left(H_{o}\right)$ and expected heterozygosity $\left(H_{e}\right)$ suggested an overall heterozygote deficit in all geographic regions, but this was strongest in the selfing samples, as might be expected with inbreeding (Additional file 1: Table S2; [54]).

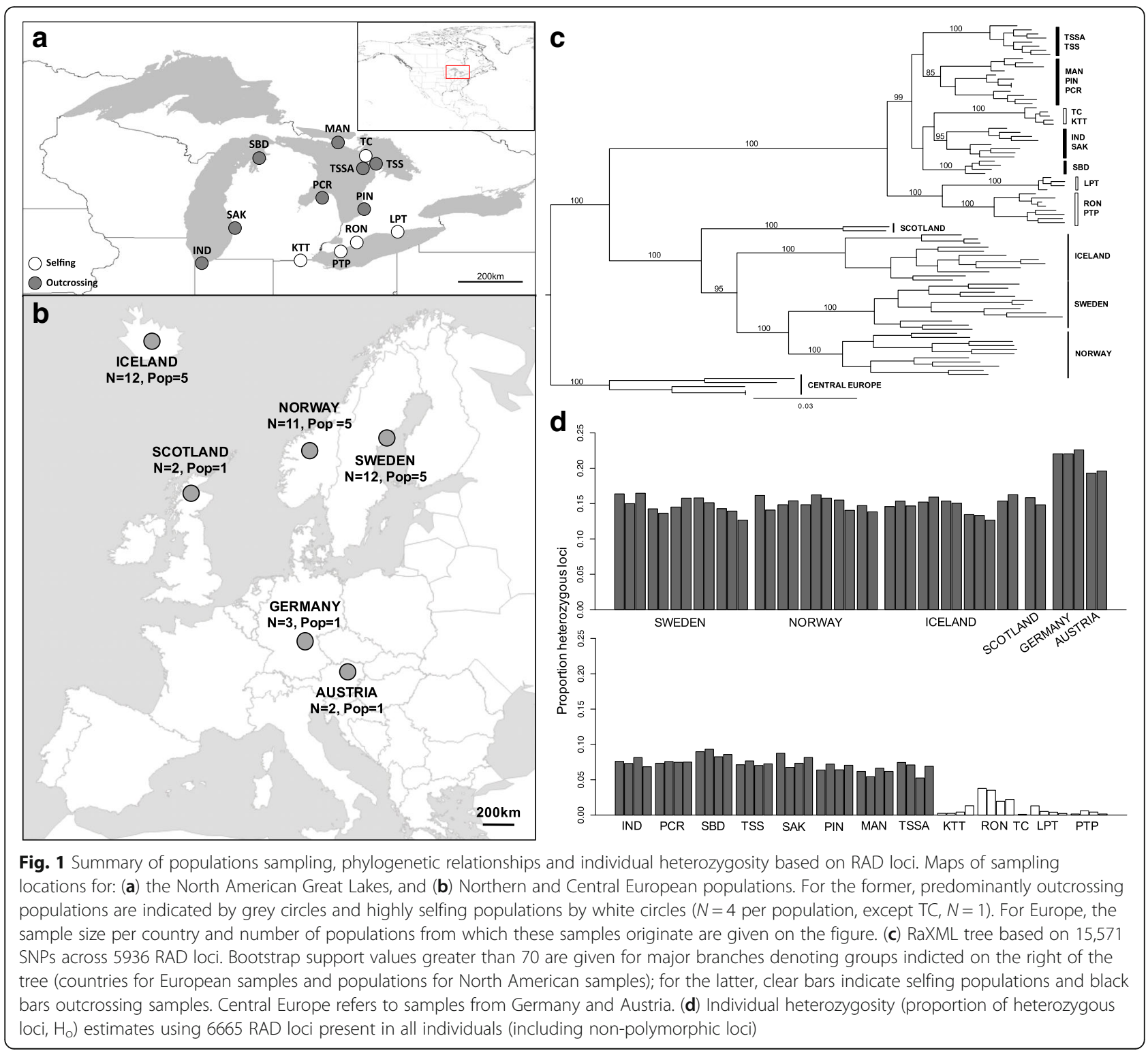


A maximum likelihood tree (RaXML) based on 15,571 SNPs (from 5936 RAD loci) shared across all samples showed strong support for separation of the two subspecies, as well as central European populations from Northern European populations (Fig. 1c), consistent with previous studies [5, 46]. Within the European (A. l. petraea) clade, individuals clustered by country of origin, with $100 \%$ bootstrap support. The North American (A. $l$. lyrata) individuals were separated into two main clusters, with individuals from selfing populations allocated to both supported clades, consistent with previous observations of multiple independent evolutionary origins of selfing in this region [54]. Additionally, outcrossing populations formed three well-supported clusters (MAN, PCR, PIN vs IND, SAK, SBD vs TSS, TSSA; Fig. 1c), again broadly consistent with previously published STRUCTURE results [54].

Principal Components Analysis confirmed the separation of Central European (EU-C) and Northern European (EU-N) groups (Additional file 1: Figure S2a), as well as clustering by country of origin across Northern Europe (Additional file 1: Figure S2b). Similar broad-scale geographic genetic structure has been observed using neutral gene sequences [46] and more recently, whole genome resequencing [5]. Even with just two or three individuals per population the PCA revealed clear genetic differentiation among most populations within Scandinavian countries (Fig. 2a-c). Furthermore, we detected significant isolation-by-distance patterns across the five populations within Norway $(\mathrm{F}=12.03, \quad p=0.050$; Additional file 1: Figure S3a) and Iceland $(\mathrm{F}=16.86, p=$ 0.018; Additional file 1: Figure S3c), and a non-significant, but positive relationship across populations within Sweden $(\mathrm{F}=2.55, p=0.1473$; Additional file 1: Figure S3b).

For the North American samples, we observed much stronger population-level PCA clustering, than previously observed using twice as many individuals genotyped at eight microsatellite markers (Fig. 3). The clustering was consistent with geographic separation of populations on lake Michigan (SBD, SAK, IND) and Erie (LPT, RON, PTP), as well as two sets of populations located on Lake Huron (TSS/TSSA and PIN/PCR). A geographically intermediate population (MAN, located on Manitoulin Island) was also intermediate in the PCA. Two geographically distinct highly selfing populations (TC and KTT) clustered together, but we could only genotype a single individual from TC. The regional sub-structuring observed using PCA and the RaXML tree might explain the absence of a significant relationship between genetic and geographic distance for these

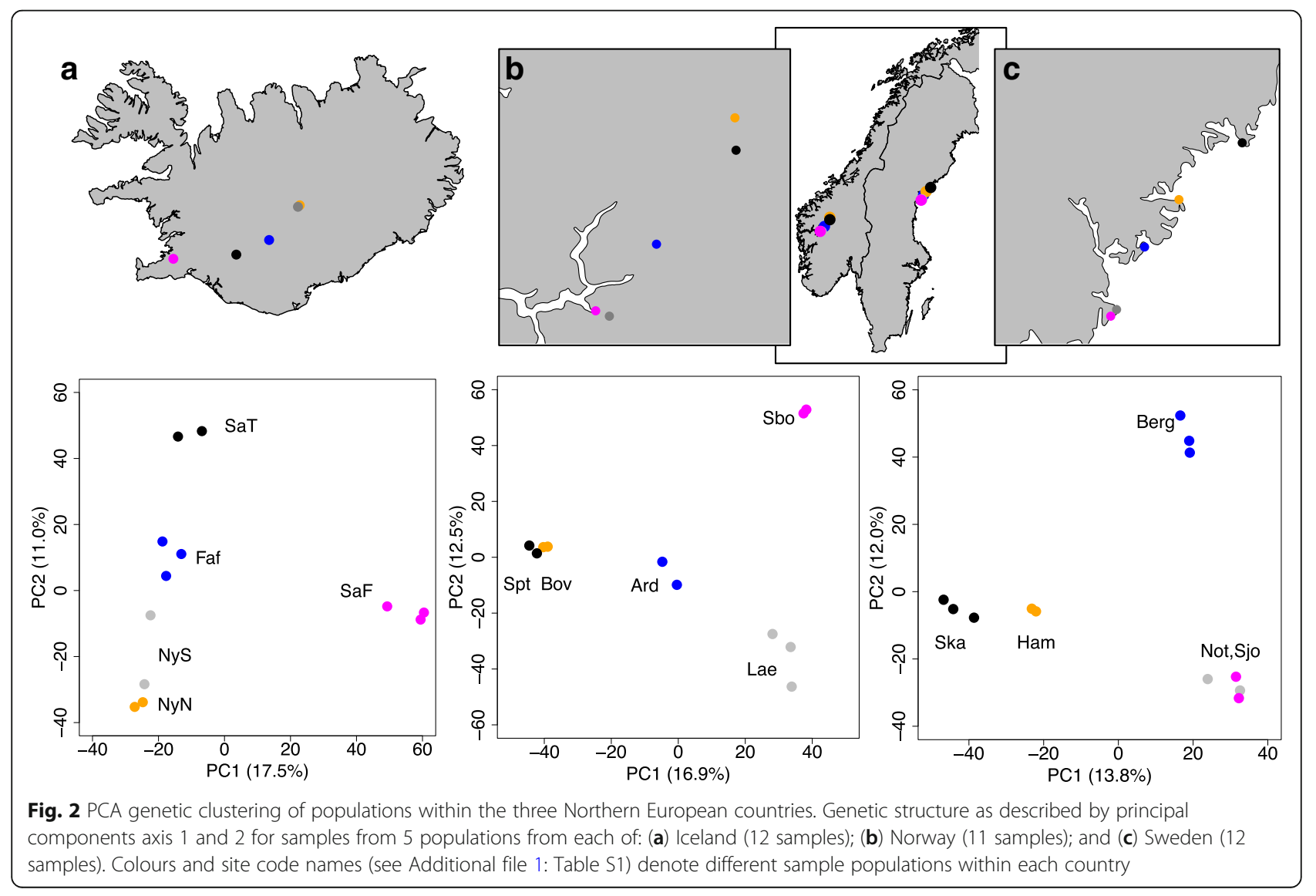



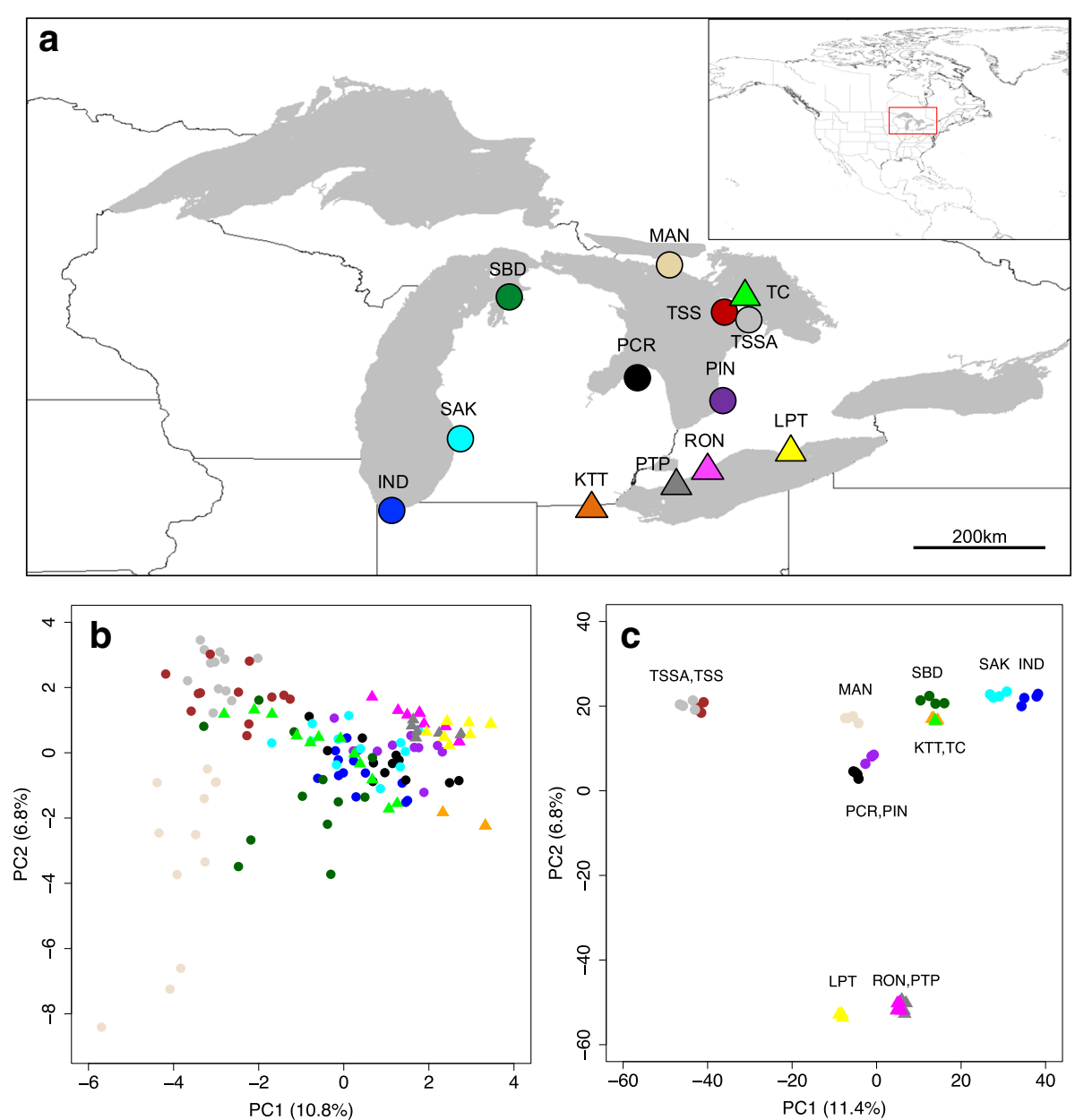

Fig. 3 Comparison of genetic clustering around the Great Lakes using RAD loci and microsatellites. The geographic location (a) of North American Great Lakes populations and their genetic clustering with respect to the first two principal component axes, using: (b) eight microsatellite loci (from Foxe et al. 2010) and (c) 3337 polymorphic RAD loci. Colours distinguish samples from different collection sites, circles represent predominantly outcrossing and triangles highly selfing populations

North American outcrossing (NA-O) populations $(\mathrm{F}=$ 1.197389, $p=0.2701$, Additional file 1: Figure S3d).

\section{Detecting signatures of balancing selection at multiple spatial scales}

\section{(A) Identifying loci showing elevated observed}

\section{heterozygosity and diversity in a low variation background}

Previous studies have used evidence of elevated diversity and observed heterozygosity in selfing or inbred populations to detect loci under balancing selection [22, 40, 57], so we predicted that genomic regions showing elevated polymorphism at RAD loci in the North American selfing (NA-S) populations might contain genes exhibiting allelic variation under balancing selection.

We first tested how varying the amount of missing data affected the density of SNPs and coverage per RAD locus, as well as summary statistics describing patterns of polymorphism and heterozygosity. Allowing for loci to be either present in all individuals ( $0 \%$ missing), or missing in up to $50 \%$ individuals ( $<50 \%$ missing), as might be expected for highly polymorphic genes that do not consistently align to the genome due to divergence from the reference (e.g. $[33,56])$, increased the number of RAD loci to 16,240 loci (2.7× higher; Additional file 1: Table S3a; Additional file 1: Figure S4). In both cases, the density of RAD reads was also reduced around the centromere of each chromosome, likely due to an increase in repetitive DNA and poor centromere assembly in the reference sequence [45]. Differences in the proportion of heterozygous loci among individuals were surprisingly consistent with or without missing data (Fig. 1d vs Additional file 1: Figure S5), suggesting that including more variable loci with missing data does not dramatically alter overall patterns of observed heterozygosity between individuals (Additional file 1: Table S2; Fig. 1d vs Additional file 1: Figure S5). However, 
including loci with missing data did slightly increase average polymorphism summary statistics (e.g. nucleotide diversity was 1.07-1.15-fold higher in each sample group when allowing for missing data; Additional file 1: Table S2), which suggests that RAD loci in more polymorphic regions of the genome were being included in the dataset. As a result of this and the higher density of RAD markers, we therefore used loci present in at least $50 \%$ individuals for analyses of other $A$. lyrata sample groups.

Using estimates of nucleotide diversity, expected heterozygosity and observed heterozygosity (proportion of heterozygous individuals) at individual RAD loci we then identified individual outlier RAD loci with the 1\% highest levels of polymorphism in the North American selfing (NA-S) group (see Additional file 1 for details). Several loci were excluded from the single outlier analyses due to uncertainty about assembly (Additional file 1: Table S4a). However, as predicted, more candidate outlier loci were identified when allowing loci to be missing in individuals than when only using those loci present in all individuals (Additional file 1: Table S4a). A BLAST search of RAD outlier sequences to a database of $A$. thaliana coding sequences (TAIR10.29) revealed four RAD loci annotated as NLR genes when allowing for up to $50 \%$ missing data, but only two using $0 \%$ missing data (Additional file 1: Table S4a,b; details in Additional file 1: results).
Genome-wide variation in nucleotide diversity and expected heterozygosity was then estimated in smoothed windows centred on polymorphic RAD loci using the programme Stacks [58], with bootstrapping used to test the significance of elevated windows of polymorphism relative to the genome-wide average. For NA-S samples the smoothed window analysis was based on $900 \mathrm{Kbp}$ windows, with 410 windows of significantly elevated diversity present in all individuals and 542 windows present in at least 50\% individuals (Table 1a). Large window sizes were necessary due to the low number of polymorphic RAD loci across NA-S samples (4327 loci present in all individuals, 7256 loci with up to $50 \%$ missing data), which limits the fine-scale resolution of this approach. Nevertheless, we predicted that Nucleotide Binding-Leucine Rich Repeat (NLR) loci (listed in Additional file 1: Table S5) would be at higher frequency in windows of significantly elevated diversity than in other smoothed windows, because they are expected to be under pathogen-mediated balancing or diversifying selection. By randomising the label of smoothed windows as 'high diversity' or 'not high diversity' we produced a null distribution of the difference in the number of NLR genes in each set of windows. We found significantly higher numbers of NLR loci in high diversity windows relative to all other windows $(p<0.0001$; Table 2a; Additional file 1: Figure S6a,b), irrespective of the filtering criteria used and despite the large window size

Table 1 High diversity genomic regions and associated disease resistance genes

\begin{tabular}{|c|c|c|c|c|c|c|c|c|}
\hline & \multicolumn{2}{|l|}{ Smoothing parameters } & \multicolumn{5}{|c|}{$\begin{array}{l}\text { Number windows showing significantly } \\
(p<0.05) \text { elevated polymorphism }\end{array}$} & \multirow[b]{2}{*}{$\begin{array}{l}\text { Proportion showing significantly } \\
\text { elevated polymorphism }\end{array}$} \\
\hline Group $^{a}$ & Proportion missing data & $\begin{array}{l}\text { Window size } \\
\text { parameter, } \sigma(\text { Kbp) }\end{array}$ & $\begin{array}{l}\text { Total number } \\
\text { of windows }\end{array}$ & $H_{e}$ & $\pi$ & $H_{e}$ and $\pi$ & Total & \\
\hline NA-S & 0 & 150 & 4327 & 151 & 165 & 94 & 410 & 0.0948 \\
\hline NA-S & $<50 \%$ & 150 & 7256 & 163 & 225 & 154 & 542 & 0.0747 \\
\hline EU-N & $<50 \%$ & 60 & 16,990 & 393 & 190 & 491 & 1074 & 0.0632 \\
\hline $\mathrm{NA}-\mathrm{O}$ & $<50 \%$ & 60 & 14,572 & 555 & 307 & 966 & 1828 & 0.1254 \\
\hline ALL-O & $<50 \%$ & 50 & 23,465 & 452 & 478 & 917 & 1847 & 0.0787 \\
\hline \multicolumn{9}{|l|}{ b) } \\
\hline Group $^{a}$ & Proportion missing data & $\begin{array}{l}\text { Number regions of } \\
\text { high diversity }\end{array}$ & $\begin{array}{l}\text { Size of regions } \\
\text { (Mbp) }\end{array}$ & \multicolumn{2}{|c|}{$\begin{array}{l}\text { Number genes } \\
\text { in regions }\end{array}$} & \multicolumn{2}{|c|}{$\begin{array}{l}\text { Number NLR genes } \\
\text { in region }\end{array}$} & Proportion of all NLR genes \\
\hline NA-S & 0 & 50 & 24.351600 & \multicolumn{2}{|c|}{4355} & \multicolumn{2}{|c|}{52} & 0.250 \\
\hline NA-S & $<50 \%$ & 43 & 22.346912 & \multicolumn{2}{|c|}{3710} & \multicolumn{2}{|c|}{49} & 0.238 \\
\hline EU-N & $<50 \%$ & 128 & 22.903806 & \multicolumn{2}{|c|}{3647} & \multicolumn{2}{|c|}{64} & 0.311 \\
\hline $\mathrm{NA}-\mathrm{O}$ & $<50 \%$ & 156 & 31.039876 & \multicolumn{2}{|c|}{5575} & \multicolumn{2}{|c|}{90} & 0.437 \\
\hline ALL-O & $<50 \%$ & 179 & 28.702652 & \multicolumn{2}{|c|}{4406} & \multicolumn{2}{|c|}{75} & 0.364 \\
\hline
\end{tabular}

(a) the number of smoothed high diversity windows with significantly elevated $H_{e}$ (expected heterozygosity), $\pi$ (nucleotide diversity) or both for different sample groups; and (b) the number of candidate NLR loci (Nucleotide binding site- Leucine rich repeat genes described in test panel in Additional file 1: Table S5) in genomic regions of significantly elevated diversity. Genomic regions are based on combining smoothed windows of significantly elevated diversity that overlap along a chromosome

${ }^{a} \mathrm{NA}-\mathrm{S}=\mathrm{N}$. American selfing, EU-N = Northern European (Sweden, Iceland and Norway), NA-O = N. American outcrossing, ALL-O = all outcrossing samples i.e. without NA-S samples 
encompassing many $A$. lyrata genes (Table 2a). For example, the start of chromosome 2, middle and end of chromosomes 7, and start of chromosome 8 showed a high density of significantly elevated diversity windows and high NLR gene density (Fig. 4a, b). By contrast, the density of another set of annotated RGAs, the LRR-RLK genes for which elevated diversity was not predicted, was not higher in windows of elevated diversity $(p=$ 0.221; Table 2b, Additional file 1: Figure S7a,b).

By merging adjacent smoothed windows of significantly elevated diversity found using loci present in all individuals, we identified 50 genomic regions of significantly elevated diversity covering $24.35 \mathrm{Mbp}(12 \%$ of chromosomes 1-8; Table 1b) and containing 4355 A. lyrata genes (14\% of all genes on chromosomes 1-8). Of these, 52 were annotated as NLR encoding genes present in our test panel (Additional file 1: Table S5) and eight exhibited variation attributed to balancing selection in other studies (summarized in Additional file 1: Table S6). Similarly, using loci with $<50 \%$ missing data, the windows of significantly elevated diversity merged into 43 genomic regions of significantly elevated diversity covering $22.35 \mathrm{Mbp}$ (11\% of total genome; Table 1a, b) and containing 3710 genes (12\% of total genes). Of these, 49 were annotated as NLR encoding genes and included three that exhibited variation attributed to balancing selection in A. thaliana or Capsella species (Additional file 1: Table S6). In contrast to the individual RAD outlier analysis, these data suggest that varying the amount of missing data does not dramatically alter the number of disease resistance candidates detected.
However, NLR encoding genes present in genomic regions of elevated diversity did not fully overlap when allowing for $0 \%$ or $<50 \%$ missing data. Of the 62 NLRs identified in total across both filtering strategies, $62.9 \%$ (39 loci) were shared between the analyses with $0 \%$ or < $50 \%$ missing data. Of these shared candidates, three genes were annotated as two $A$. thaliana NLRs, specifically At4g16950 (RPS2: [59]) and At5g63020 (no known function), both with prior evidence for balancing selection (Additional file 1: Additional file 1: Table S6; genomic position indicated in Fig. 4). Interestingly, three of the four candidate genes identified by the single outlier analyses in the NA-S comparison were also found in smoothed genomic regions of elevated diversity (Additional file 1: Table S4b), in addition to many new candidate NLRs. We therefore adopted only the smoothing approach for the remaining analyses.

\section{(B) Identifying loci showing elevated diversity, reduced genetic differentiation and intermediate allele frequencies in outcrossing populations}

Balancing selection may also leave a signature of reduced genetic differentiation among populations, as well as shifts in the allele frequency spectrum towards intermediate allele frequencies [22, 23]. These can be observed, respectively, by lower estimates of genetic differentiation, $F_{S T}$, among populations relative to the genome-wide average, and elevated Tajima's D relative to the genome-wide average. We used 35 outcrossing individuals of $A$. lyrata from 15 populations (originating from three countries) in Northern Europe (EU-N) and 32 individuals from eight outcrossing populations

Table 2 Elevated density of disease resistance candidates in areas of high genomic polymorphism

\begin{tabular}{|c|c|c|c|c|}
\hline \multicolumn{2}{|l|}{ a) } & \multicolumn{3}{|l|}{ Mean number NLR genes } \\
\hline Group $^{a}$ & Proportion missing data & All high diversity windows & All other windows & Significance of difference \\
\hline NA-S & 0 & 0.615 & 0.299 & $* * *$ \\
\hline NA-S & $<50 \%$ & 0.738 & 0.332 & $* * *$ \\
\hline EU-N & $<50 \%$ & 0.331 & 0.111 & $* * *$ \\
\hline $\mathrm{NA}-\mathrm{O}$ & $<50 \%$ & 0.402 & 0.102 & $* * *$ \\
\hline ALL-O & $<50 \%$ & 0.332 & 0.080 & $* * *$ \\
\hline b) & & \multicolumn{3}{|l|}{ Mean number LRR-RLK genes } \\
\hline Group $^{a}$ & Proportion missing data & All high diversity windows & All other windows & Significance of difference \\
\hline NA-S & 0 & 0.390 & 0.486 & * \\
\hline NA-S & $<50 \%$ & 0.443 & 0.491 & ns \\
\hline EU-N & $<50 \%$ & 0.156 & 0.206 & * \\
\hline $\mathrm{NA}-\mathrm{O}$ & $<50 \%$ & 0.172 & 0.194 & ns \\
\hline ALL-O & $<50 \%$ & 0.140 & 0.171 & * \\
\hline
\end{tabular}

(a) The average number of NLR genes and (b) LRR-RLK genes in significantly elevated diversity windows and all other windows are given, along with the significance of the difference in average number of genes per window based on a randomisation test. These averages are based on numbers of genes present in individual smoothed windows defined by Stacks and significance of the difference is denoted as ${ }^{* *} p<0.0001, * * 0<0.001,{ }^{*} p<0.01, \mathrm{~ns}=p>0.05$

${ }^{a} \mathrm{NA}-\mathrm{S}=\mathrm{N}$. American selfing, EU-N $=$ Northern European (Sweden, Iceland and Norway), NA-O = N. American outcrossing, ALL-O = all outcrossing samples i.e. without NA-S samples 


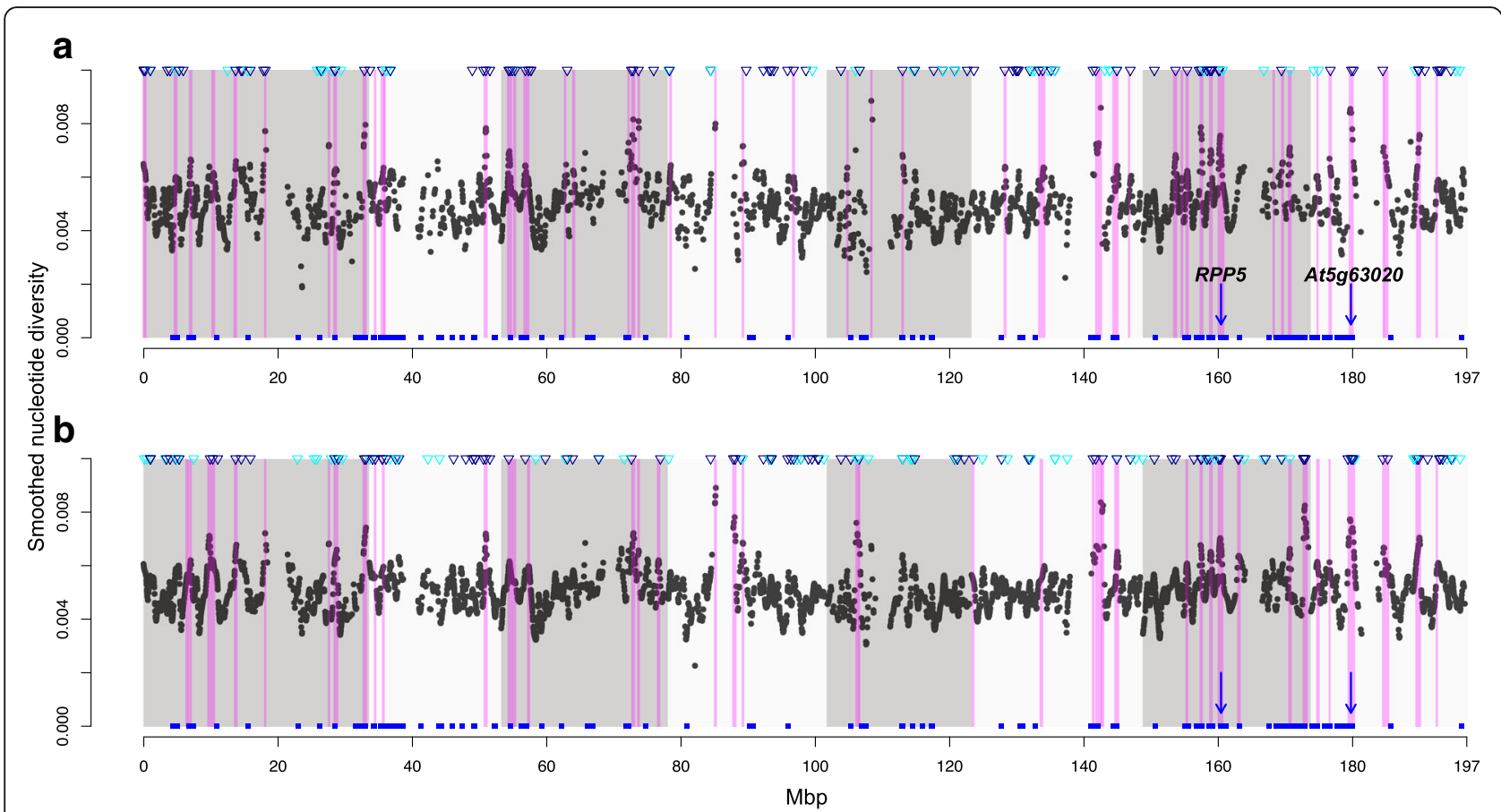

Fig. 4 Genomic regions of elevated polymorphism and location of NLR genes across North American selfing populations. Smoothed nucleotide diversity plots based on (a) 4327 loci present in all, or (b) 7256 loci present in at least 50\% of individuals. Regions of significantly elevated nucleotide and/or gene diversity (neighbouring windows, $450 \mathrm{kbp}$ either side of a polymorphic locus; $p<0.05$ ) are marked in purple, with the eight chromosomes shaded alternately in dark and light grey. The genomic locations of single outlier loci are marked along the top of the graph by downward pointing blue triangles (dark blue = single outlier based on nucleotide and gene diversity, or turquoise = based on observed heterozygosity). The location of 206 annotated NLR genes on the A. lyrata reference chromosomes 1-8 are denoted by blue squares, with two candidates (RPP5 [95] and At5g63020 [40]) marked by downward pointing blue arrows. These annotated candidates are present in high diversity regions for both sets of RAD loci and show a priori evidence for balancing selection in A. thaliana (Additional file 1: Table S6)

around the North American Great Lakes (NA-O), for which genetic structure among populations within each region is strong (Fig. 3, Additional file 1: Figure S2b). Specifically, we identified all genomic windows showing both significantly elevated diversity and either signatures of reduced $\mathrm{F}_{\mathrm{ST}}$ or positive Tajima's $\mathrm{D}$ (Additional file 1: Table S7) using 16,990 polymorphic loci present in at least $50 \%$ of EU-N samples and 14,572 polymorphic loci present in at least $50 \%$ of NA-O samples. The number of polymorphic RAD loci is notably lower than the 64,056 predicted using the Pst1 enzyme, partly because one third of loci are not being assembled in Stacks (e.g. reads stochastically absent from a sample, or not aligned singly to the genome), but mostly due to the requirements for loci to be present in no fewer than $50 \%$ individuals in a sample group (see methods for details). Using loci present in fewer than $50 \%$ of individuals in a sampling group increased the density of loci, but also resulted in low sample sizes to estimate diversity and differentiation at individual loci, which we wanted to avoid.

First, we identified smoothed windows of significantly elevated diversity $\left(\mathrm{H}_{\mathrm{e}}\right.$ and/or $\pi$ ) for EU-N and NA-O samples separately using $300 \mathrm{Kbp}$ windows centred on polymorphic RAD loci. This is a broad window size, but given that there were polymorphic RAD loci on average every $11.595 \mathrm{Kbp}$ for NA-O samples and $13.519 \mathrm{Kbp}$ for EU-N samples, and Stacks estimates average window polymorphism with reduced weight on RAD loci further from the centre of the window, we are confident that the effective resolution of our windows was more localised than the total window size suggests. Generally, there was some overlap in the location of high diversity windows in the EU-N and NA-O samples (Additional file 1: Figure S8a vs S8b), although the proportion of windows showing significantly elevated diversity was higher (12.5\%) for the NA-O samples than for the EU-N samples $(6.3 \%$, Table 1a), likely due to the higher overall diversity across the EU-N samples.

As with the selfing samples, NLR-encoding genes were found at significantly higher densities in smoothed genomic windows of significantly elevated diversity relative to all other windows (randomisation test: $p<0.0001$ for both EU-N and NA-O; Table 2b; Additional file 1: Figure S6c,d). By contrast, LRR-RLK genes were either at higher frequency in smoothed windows of non-elevated diversity or showed no significant difference (EU-N: $p=$ 0.004, NA-O: $p=0.067$, Table $2 \mathrm{~b}$, Additional file 1: Figure $S 7 c, d)$. When individual windows were merged into 
larger genomic regions of elevated diversity, there were on average 2.8 and 2.9 NLRs per Mbp in regions of significantly elevated diversity for EU-N and NA-O samples respectively (compared to a genome-wide average of 1.0 NLR per Mbp). A total of 31 NLR genes were found to be in high diversity genomic regions in both sample groups (Fig. 5; Additional file 1: Figure S8), including eight genes showing evidence for balancing selection in other studies (Additional file 1: Table S6). However, just 12 NLRs $(9.6 \%$ of all NLRs identified; Fig. 5a, b) were found to be present in high diversity genomic regions in all three sample groups: EU-N, NA-O and NA-S. Interestingly, none of these loci have yet been described as showing signatures of balancing selection in other species.

To attempt to distinguish balancing from other types of selection, we then identified smoothed windows showing reduced $\mathrm{F}_{\mathrm{ST}}$ between geographic regions (EU-N or NA-O) or positive Tajima's D (suggesting shifts towards intermediate allele frequencies) within geographic regions that overlapped with smoothed windows of significantly elevated diversity in one or both sample groups. For the EU-N group, just five smoothed windows of significantly elevated diversity (out of 1074 in total) showed $\mathrm{F}_{\mathrm{ST}}$ values in the bottom $5 \%$ of windows (Additional file 1: Figure S10a). These windows merged into three genomic regions that together were $0.36 \mathrm{Mbp}$ in length and contained 26 genes, but no NLR genes. For the NA-O group only 11 windows of significantly elevated diversity (out of 1828 in total) had $\mathrm{F}_{\mathrm{ST}}$ estimates in the bottom $5 \%$ of all windows (Fig. 6a). These windows merged into two genomic regions that were $0.20 \mathrm{Mbp}$ and $0.12 \mathrm{Mbp}$ in length and respectively contained 19 and 17 genes, of which two were NLRs (both on Chromosome 6) involved in interactions with oomycete pathogens in A. thaliana: At2g14080 (RPP28; Sepahvand and Holub, unpublished) and At3g44630 (RPP1 cluster gene; [39]).
Interestingly, a high proportion of windows of significantly elevated diversity overlapped with windows of high genetic differentiation (in the top $5 \% \mathrm{~F}_{\mathrm{ST}}$ values) across both EU-N countries (Additional file 1: Figure S10a) and NA-O genetic clusters (Fig. 6a). For EU-N samples, there were 137 overlapping windows (3.804Mbp in length) containing 601 genes, of which nine were NLRs (summarised in Additional file 1: Table S7), and six showed signatures of balancing selection in previous studies (Additional file 1: Table S6). Two loci were annotated as At1g63730 (uncharacterised resistance function), another two were annotated as At1g63870 (RLM-B homolog involved in fungal resistance; Staal et al. 2006), and the final two were both annotated as At5g43470 (RPP8, involved in oomycete resistance; McDowell et al. 1998). For NA-O samples, there were 182 overlapping windows of high diversity and $\mathrm{F}_{\mathrm{ST}}$ (5.17Mbp in length) containing 791 genes, of which five were annotated as NLR encoding genes (Additional file 1: Table S7). One locus was annotated as At1g50180 (uncharacterised resistance function), two as At3g44400 (RPP1 cluster; [39]) and a final locus as At4g11340 (uncharacterised resistance function).

Finally, we identified smoothed windows showing significantly elevated diversity that overlapped with 120 Kbp sliding windows showing elevated Tajima's D. To minimise the confounding effects of demographic history across countries, we identified the windows with the $5 \%$ highest values of Tajima's D for each Northern European country separately (Norway, Sweden and Iceland) and for all NA-O samples combined. The average value across all SNPs for Northern European countries was consistently positive (Norway $\mathrm{D}=0.547$, Sweden $\mathrm{D}=0.481$, Iceland $\mathrm{D}=0.722$; Additional file 1 : Figure S9b-d), but for NA-O was negative (-0.395; Additional file 1: Figure S9a).

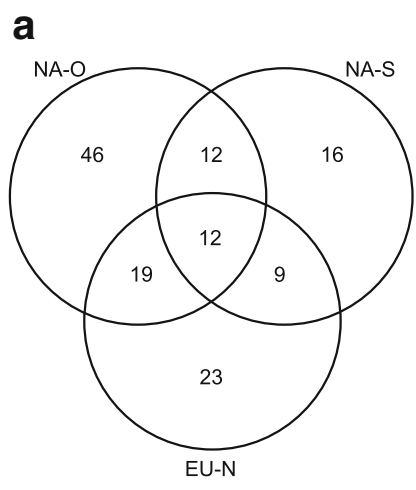

b
\begin{tabular}{|c|c|c|c|}
\hline $\begin{array}{c}\text { A. lyrata } \\
\text { gene ID }\end{array}$ & $\begin{array}{c}\text { A. lyrata } \\
\text { chromosome }\end{array}$ & $\begin{array}{c}\text { A. thaliana } \\
\text { annotation }\end{array}$ & $\begin{array}{c}\text { Disease resistance } \\
\text { protein }\end{array}$ \\
\hline 915002 & 1 & AT $3 G 44400$ & TIR-NBS-LRR \\
\hline 915006 & 1 & AT3G44400 & TIR-NBS-LRR \\
\hline 891930 & 7 & AT5G38340 & TIR-NBS-LRR \\
\hline 891927 & 7 & AT5G 38350 & NBS-LRR \\
\hline 891933 & 7 & AT5G38350 & NBS-LRR \\
\hline 915426 & 8 & AT5G46260 & TIR-NBS-LRR \\
\hline 915424 & 8 & AT5G46270 & TIR-NBS-LRR \\
\hline 915427 & 8 & AT5G46270 & TIR-NBS-LRR \\
\hline 915422 & 8 & AT5G46450 & TIR-NBS-LRR \\
\hline 915423 & 8 & AT5G46450 & TIR-NBS-LRR \\
\hline 915425 & 8 & AT5G46450 & TIR-NBS-LRR \\
\hline 494325 & 8 & AT5G46470 & TIR-NBS-LRR \\
\hline
\end{tabular}

Fig. 5 Disease resistance loci in genomic regions of significantly elevated diversity shared between sample groups. a Venn diagram illustrating the number of candidate A. lyrata genes unique to and shared between different groups (NA-O, NA-S and EU-N sample groups). This analysis is based on RAD loci with up to 50\% individuals missing within each group; (b) a list of the 12 annotated NLR genes that were shared between all groups. Note that some A. lyrata genes have the same TAIR annotations for A. thaliana (see highlighted sections) 


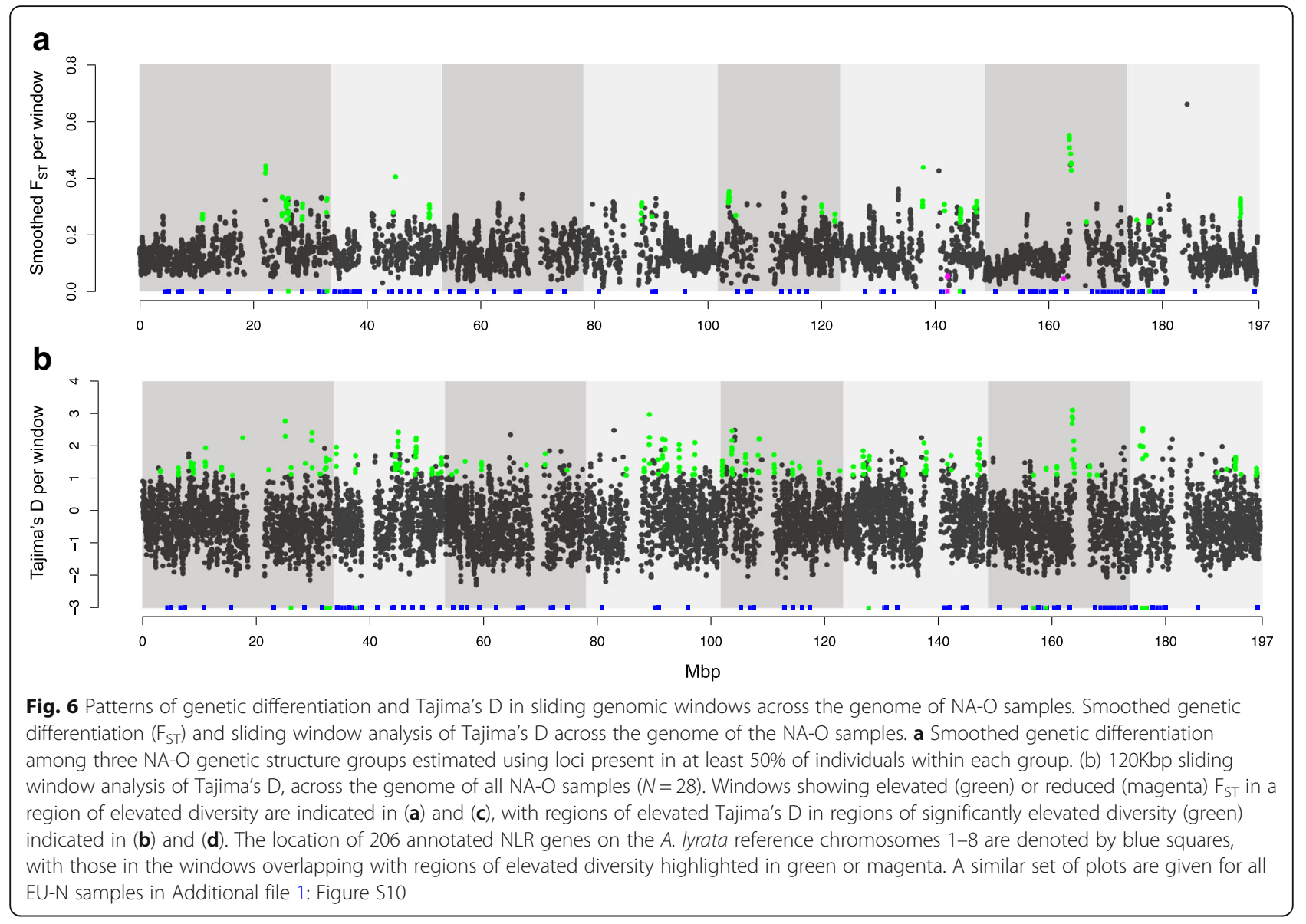

Interestingly, approximately $25 \%$ of the smoothed windows of elevated Tajima's D also overlapped with smoothed windows of significantly elevated diversity in each Northern European country (Norway $=25.3 \%$, Sweden $=29.3 \%$, Iceland $=23.8 \%$; Table 3; Additional file 1: Figure S10b,c,d). NLR encoding genes were identified in these windows, ranging from five in Sweden to 12 in Norway (Table 3). Most of the NLR candidates were unique to one country, with just four NLRs identified in overlapping windows in more than one country (Table 3 ).
One A. lyrata NLR-gene on chromosome 7 (annotated as At5g38850) was identified in all three Scandinavian (EU-N) countries, although to our knowledge no functional resistance allele has been described in A. thaliana (Additional file 1: Table S7).

For the NA-O sample set, $58.1 \%$ of windows of elevated Tajima's D overlapped with windows of significantly elevated diversity (Table 3; Fig. 6b), and a greater number of known NLRs were identified in these overlapping regions compared to the Scandinavian samples (33

Table 3 Numbers of overlapping windows of elevated diversity and D for different geographic regions

\begin{tabular}{|c|c|c|c|c|}
\hline & \multicolumn{3}{|l|}{ EU-N ${ }^{a}$} & \multirow{2}{*}{$\begin{array}{l}N A-O^{b} \\
(N=32)\end{array}$} \\
\hline & Norway $(N=11)$ & Sweden $(N=12)$ & Iceland ( $N=12)$ & \\
\hline Total windows & 13,679 & 13,650 & 13,607 & 14,278 \\
\hline High Tajima's D and high diversity overlap & 173 & 200 & 162 & 415 \\
\hline Number A. lyrata genes in overlap & 904 & 1060 & 883 & 2321 \\
\hline Number NLR genes in overlap & 12 & 5 & 8 & 33 \\
\hline Number country-specific NLR genes & 9 & 2 & 5 & - \\
\hline
\end{tabular}

The total number of windows in which Tajima's D could be estimated (i.e. contained a SNP) is given for each of the Northern European countries (Norway, Sweden, Iceland) and all North American outcrossing samples. The overlap between windows with elevated Tajima's D (top 5\% of all windows) and significantly elevated diversity is also given, as well as the total number of predicted $A$. lyrata genes and genes annotated as NLR loci in the overlapping high Tajima's D and high diversity windows. For the Northern European samples, the number of NLR genes identified in overlapping genomic regions in just one country

${ }^{\mathrm{a}}$ Northern European countries, ${ }^{\mathrm{b}} \mathrm{N}$. American outcrossing 
putative NLR encoding genes in total). Of particular interest were three NLR-genes (two annotated as At1g56540, and another annotated as At1g59780) that also show elevated polymorphism and high Tajima's D in A. thaliana (Additional file 1: Table S6). Both are located in gene clusters associated with resistance to oomycete pathogens in A. thaliana [40,60].

To potentially increase power for detecting signatures of reduced genetic differentiation we also assessed evidence for elevated diversity windows across all outcrossing samples (NA-O, EU-N and including central European and Scottish samples). In total 1847 windows showed significantly elevated $\mathrm{H}_{\mathrm{e}}$ and/or $\pi$, which is higher than that observed for the individual sample groups (NA-O, EU-N and NA-S). NLRs were again significantly overrepresented in windows of elevated diversity $(p<0.0001$; Table 1b; Additional file 1: Figure S6e), whereas LRR-RLK genes were at slightly higher frequency in windows of non-elevated diversity $(p=0.01$, Table 2b, Additional file 1: Figure S7e). These windows merged into 179 genomic regions (28.7 Mbp in length) of elevated diversity distributed across the genome (Additional file 1: Figure S11a). Of the 4406 genes in these regions, 75 were NLR-encoding genes with 10 loci annotated as $A$. thaliana resistance genes that have been described as under selection in studies of either A. thaliana or Capsella species (Additional file 1: Table S6), including genes annotated as RPP1, RPP13, RPP7 and a paralog of WRR4 (Additional file 1: Table S6).

On average, smoothed $\mathrm{F}_{\mathrm{ST}}$ between the two subspecies was 0.278 (Additional file 1: Figure S11b), which should be a sufficiently high baseline for detecting windows of reduced genetic differentiation that overlap with windows of elevated diversity. However, only one elevated diversity window on chromosome 7 also displayed a smoothed $\mathrm{F}_{\mathrm{ST}}$ value in the bottom $5 \%$ of all windows (highlighted in pink in Additional file 1: Figure S11b). Interestingly, this window contained two NLR encoding genes annotated as At5g40090 and At5g40100, although to our knowledge there are no prior reports of these loci being involved in interactions with pathogens (Additional file 1: Table S7). As observed for the EU-N and NA-O groups, the overlap between windows of significantly elevated diversity and those with $\mathrm{F}_{\mathrm{ST}}$ values in the top $5 \%$ of all windows was much higher (Additional file 1: Figure S11b). Of 494 overlapping windows (11.66 Mbp in length) there were 12 NLR-encoding genes, most of which represent NLR genes with uncharacterised functions in $A$. thaliana (summarised in Additional file 1: Table S7).

\section{Discussion}

In this study we demonstrate that high throughput genotyping of a small number of individuals across a large number of populations can identify signatures of different forms of selection. Specifically, we identified genome-wide signatures of balancing and diversifying selection within and between two subspecies of $A$. lyrata, and then focused on whether annotated disease resistance genes predicted to be under pathogen-mediated selection were over-represented in genomic regions showing elevated polymorphism. Our results reveal three key findings: (1) we confirmed previously observed patterns of genetic diversity and genetic structure across the subspecies of $A$. lyrata, but also revealed finer-scale spatial genetic structure within countries using only several individuals per population; (2) genomic regions showing elevated polymorphism contained a significantly higher number of disease resistance genes than other genomic windows; and (3) relatively few genomic regions showed both elevated diversity and reduced genetic differentiation among sample groups, a key signature distinguishing balancing from other forms of selection.

\section{Spatially arranged genetic structure, diversity and heterozygosity across multiple spatial scales in A. lyrata}

Previous studies have shown strong population structure across the range of $A$. lyrata with the origin of diversity in Central Europe [5, 48, 49], a bottleneck in North American populations [46], and additional bottlenecks and independent origins of North American selfing populations [54, 56]. Using only $2-4$ individuals per population, but multiple populations per region and analyses based on RAD loci, we found remarkably similar results to those based on whole genome re-sequencing of fewer populations per region but more individuals per population (e.g. [5]). For example, we observed the separation of a North European and Eurasian/Amphiberingean genepool from a genetically highly diverse Central European genepool [61, 62], which was estimated based on whole chloroplast genome sequence data to have occurred approximately 500,000 years ago [16]. This northern genepool must have undergone severe Pleistocenic differentiation and multiple range expansion and contractions, as also indicated by complex reticulate and cross-species evolutionary processes in the Russian Far East and the amphiberingean area [61-63]. Interestingly, we could also clearly differentiate most populations within the three Scandinavian countries (Fig. 2) and found evidence for isolation by distance within countries (Additional file 1: Figure S3), suggesting that genetic differentiation among populations within regions is relatively strong. Similarly, we found stronger genetic differentiation among the $A$. l. lyrata populations around the Great Lakes than observed using microsatellites (Fig. 3b, c), even though we only genotyped half the number of individuals per population with RAD-seq. The 
RAD markers thus clearly resolved population genetic structure within and among species to set a framework for interpreting patterns of selection.

\section{Detecting signatures of selection at multiple spatial scales The effects of strategies for filtering and outlier detection}

Overall patterns of polymorphism and heterozygosity were not substantially affected by filtering to allow inclusion of RAD loci that were not present in all individuals (Additional file 1: Table S2). This is consistent with recent work showing that varying the amount of missing data had limited effects on summary statistics estimated using empirical and simulated data [64]. In our study, relaxed filtering to consider loci present in at least $50 \%$ individuals had limited effect on the power to detect regions under balancing selection compared to requiring loci to be present in all individuals (Table 1b), but resulted in higher nucleotide diversity and expected heterozygosity (Additional file 1: Table S2), suggesting inclusion of more polymorphic gene regions.

Consistent with other studies, our results suggest that outlier analyses based on genomic smoothing are preferable to using single RAD loci when looking for genes showing elevated polymorphism. For example, using RAD-seq data and the Stacks smoothing algorithm, Hohlenlohe et al. (2010) similarly found evidence for high diversity and reduced $\mathrm{F}_{\mathrm{ST}}$ at the stickleback Major Histocompatibility locus, a locus known to show signatures of balancing selection in a wide range of taxa. Using smoothing over multiple RAD loci can minimise the risk of identifying high diversity genomic regions based on an incorrectly assembled individual locus and thus increase the power to distinguish outliers. Additionally, given the importance of accurately calling SNPs for assessing variation in diversity, we used a $10 \times$ minimum coverage per locus for calling heterozygotes and used the rxstacks module from the Stacks pipeline (http:// catchenlab.life.illinois.edu/stacks/comp-v1/rxstacks.php) to exclude and, where possible, correct likely sequencing errors from our RAD dataset. Finally, our study demonstrates the power of this smoothing approach even with relatively sparse marker density.

\section{Disease resistance candidates are associated with genomic regions of elevated diversity}

Nucleotide binding site-leucine rich repeat proteins (NLRs), are a major class of Resistance Gene Analogs (RGA) known to be good at generating allelic diversity, and such diversity is important for recognizing a diverse pool of potential pathogen effectors [39, 65]. NLRs are a well-annotated gene family in A. thaliana and around two-thirds of known $R$-genes in $A$. thaliana have a matching gene in A. lyrata. We found, as predicted, that the density of NLR loci in A. lyrata was significantly higher in genomic regions of elevated allelic diversity than other regions of the genome and that this result was consistent across different sample groups of $\mathrm{A}$. lyrata (Table 2). By contrast, the density of a related set of RGAs, LRR-SLKs, was not higher in regions of elevated diversity, as might be expected from their involvement in more conserved ligand interactions [35]. This is consistent with whole genome sequencing studies, with the NLR class of disease resistance genes in A. thaliana found to be at higher frequency in regions of elevated diversity and SLKs proportionately less well-represented in high diversity regions [38]. Similarly, using shotgun sequence fragments, Cork \& Purugganan (2005) [66] identified genomic regions of high synonymous diversity across $A$. thaliana ecotypes, and found one containing a predicted TIR-NBS-LRR gene.

An advantage of our broad sampling of $A$. lyrata subspecies was that we could identify candidate loci present in high diversity genomic regions in multiple independent sample groups. In total, $37.9 \%$ of the 137 NLRs were present in high diversity regions in at least two sample groups, and $8.8 \%$ across all three sample groups (Fig. 5). Furthermore, NLR genes shared between North American and European A. lyrata included disease resistance candidates known to show elevated levels of polymorphism in A. thaliana (Additional file 1: Table S6); for example, RPP7 and RPP13 involved in strain specific resistance to the downy mildew pathogen Hyaloperonospora arabidopsidis [40,67], and a homolog of RLM1B involved in fungal resistance $[40,68]$. Interestingly, one gene was annotated as a paralog of WRR4 (At1g56540) and in the same cluster as WRR4 (At1g56510) [60]. WRR4 confers broad spectrum resistance to the oomycete rust pathogen Albugo candida was in a genomic region of elevated diversity in all outcrossing groups in our study, and also shows elevated diversity in both $A$. thaliana [40] and Capsella sp. [57]. This locus showed higher nucleotide diversity based on Sanger sequencing for a more extensive sampling of $A$. l. lyrata from the Great Lakes region when compared to average nucleotide diversity across a conservative set of RAD loci present in all individuals [51]. Several other $R$-genes thought to be influenced by balancing selection were only present in genomic regions of significantly elevated diversity in one of the population groups; for example, two genes recognising Pseudomonas bacteria in the NA-O samples, RPM1 [69, 70] and RPS2 $[59,71]$. This might not be unexpected since these are members of large gene families where there might be variation in the number of copies retained between populations [42]. Nevertheless, together with our results, these studies support the hypothesis that the evolution of NLR genes may be driving patterns of elevated polymorphism in some parts of the genome. 
One criticism of the use of RAD markers is that the resolution of such loci to detect individual genes under selection is low, particularly when a gene has been under long-term selection and recombination has reduced linkage to surrounding sequences [18]. By reducing the number of individuals a locus had to be present in, or reducing the read coverage per locus, we could have increased marker density, but at the cost of reduced accuracy in estimating certain statistics across the genome. For example, low sample sizes are a particular problem for estimating $\mathrm{F}_{\mathrm{ST}}$. In any case, the relatively high density of individual markers, particularly in the more variable outcrossing sample groups (on average every $11.6 \mathrm{Kbp}$ or $13.5 \mathrm{Kbp}$ ), means that our resolution with respect to individual genes was relatively high. For example, even for the NA-S samples, individual high diversity individual RAD loci could be identified in the vicinity $(+/-$ $2500 \mathrm{bp}$ ) of annotated $R$-genes (see NA-S single outlier overlap with smoothed outlier windows; Additional file 1: Table S4).

Our study also shows the potential for using genome scan data in a reverse genetics approach to identify loci in A. lyrata that may be generating allelic diversity for disease resistance. Targeted sequencing of candidate genes would of course be necessary to confirm the elevated patterns of diversity, and gene knock-outs of the corresponding $A$. lyrata loci could be used to see whether this reduces fitness under field conditions, in particluar when associated with elevated densities of pathogenic microbes. Most of the $R$-genes we focus on in this paper are examples from $A$. thaliana that have had reports of a functional allele conferring resistance to a bacterial, fungal or oomycete pathogen (Additional file 1: Table S6, S7). However, we also identify NLR loci for which there is no evidence in other studies for an interaction with a pathogen (Additional file 1: Table S6/S7), making these interesting novel candidates for further study.

\section{Multiple population genetic statistics can help detect genome-wide signatures of balancing selection}

In addition to elevated polymorphism, balancing selection may also leave signatures of reduced genetic differentiation among populations and shifts in the allele frequency spectrum towards intermediate frequencies $[22,23]$. A key aim of our broad sampling approach was to ensure high neutral $\mathrm{F}_{\mathrm{ST}}$ among sample groups and therefore increase our power to detect this signature of balancing selection (e.g. [24]). However, we found relatively few genomic regions (and associated NLR candidates) showing both elevated diversity and reduced $\mathrm{F}_{\mathrm{ST}}$ among diverged sample groups, with relatively more genomic regions showing both elevated polymorphism and elevated $\mathrm{F}_{\mathrm{ST}}$ (Fig. 6; Additional file 1: Figure S10; Additional file 1: Table S7). This is similar to recent results from a study on Anopheles mosquitos using a comparable strategy to our own (Kamdem et al. (2017) [4]. In their case, the limited evidence for balancing selection could result from low levels of background $\mathrm{F}_{\mathrm{ST}}$ within the two mosquito species compared, but our sampling of a small number of individuals from strongly differentiated populations suggests that the rarity of these signatures of balancing selection could be more than just lack of power to detect outliers. A genome-wide screen of a population of Drosophila melanogaster also only found 30 of 13,900 protein-coding genes to show signatures of balancing selection based on elevated Tajima's D and Watterson's estimator $\varnothing_{\mathrm{W}}$ [17]. Thus, balancing selection might be a relatively rare form of selection shaping genome-wide patterns of polymorphism.

The higher concordance between elevated diversity and elevated $\mathrm{F}_{\mathrm{ST}}$ could represent the random fixation of diverged NLR alleles in the different subspecies by genetic drift, or selection maintaining diverged alleles in different geographic locations. Selection by distinct pathogen communities in different geographic locations could result in increased differentiation between geographic regions or populations, and also elevate diversity across sample groups at these loci [72]. Such patterns of diversifying selection have been described for MHC class II genes in amphibians (e.g. [73]), as well as at effector proteins that interact with target host plant defense proteins in the stem rust fungus, Puccinia graminis [74]. However, despite high allelic divergence and diversity at NLR genes in plants, there is limited evidence for this translating into increased divergence among natural populations [40]. Here, we identify a number of interesting candidate loci in regions of the genome showing signatures of diversifying selection. For example a downy mildew resistance locus RPP8, showed elevated polymorphism, $\mathrm{F}_{\mathrm{ST}}$ and Tajima's D across EU-N countries (Additional file 1: Table S7). In A. thaliana, RPP8 also shows elevated levels of polymorphism [71, 75] and a long evolutionary history consistent with long-term balancing selection [39, 76]. However, many candidate NLR genes identified in our study have not yet been functionally described in A. thaliana (Additional file 1: Table S7) and would require further functional testing.

In contrast to lack of evidence for reduced population structure in regions of elevated diversity, there was a large degree of overlap between genomic regions of significantly elevated polymorphism and elevated Tajima's $\mathrm{D}$ (shifts to intermediate allele frequencies). For example, a $R P P 8$ homolog and a paralog of WRR4 were present in such regions, along with genes annotated as part of the RPP7 and RPP1 gene clusters (Additional file 1: Table S7). Specifically, the WRR4 paralog showed elevated diversity in both EU-N and NA-O sample groups, as well as 
elevated D across the NA-O group and in Norweigian samples. Interestingly, both this WRR4 paralog and At1g59780 (part of the RPP7 gene cluster [67]), show elevated diversity and high Tajima's D in A. thaliana [40]. Furthermore, the WRR4 paralog exhibits signatures of balancing selection, high diversity and reduced $\mathrm{F}_{\mathrm{ST}}$, in both Capsella grandiflora and C. rubella [57]. Thus, although the number of loci resolved was low, detection of genes under balancing selection may be stronger based on shifts in allele frequency spectra than reduced differentiation. However, it should be noted that such an overlap between elevated polymorphism and Tajima's D could partly result from the non-independence of these statistics, but also from the presence of fine-scale genetic structure within our sample groups [25]. Our strategy to sample broadly across populations rather than intensively within populations while useful for detecting population structure and increased polymorphism might not have been as appropriate for detecting shifts in allele frequency spectra due to selection rather than demography.

\section{Conclusions}

By sampling a small number of individuals within populations across multiple spatial scales, we confirmed and extended previous predictions about both broad- and fine-scale phylogeographic differentiation in A. lyrata. Our smoothed outlier analyses based on relaxed filtering of missing data demonstrated that high throughput genotyping approaches, such as RAD-seq, retain power to detect genes in regions of the genome showing signatures of different types of selection. We identified genomic regions of elevated polymorphism containing disease resistance candidates known to be influenced by balancing selection in $A$. thaliana. Furthermore, within regions showing classical signatures of balancing selection (high diversity, low differentiation or intermediate allele frequencies), we identified novel candidate disease resistance genes, which have not been previously described as balanced polymorphisms in other taxa. Interestingly, we identified relatively few signatures of classical balancing selection, specifically genomic regions showing both high diversity and reduced differentiation. While this could result from a lack of power to detect reduced $\mathrm{F}_{\mathrm{ST}}$ with $\mathrm{RAD}$ markers, the high average differentiation between and within A. lyrata subspecies instead suggests that this mode of selection could be relatively rare, even for a priori candidates such as disease resistance loci. In fact, candidate disease resistance loci in A. lyrata were mostly located in regions showing both elevated polymorphism and elevated differentiation, suggesting that diversifying selection may play a more important role than expected in pathogen resistance evolution across broad geographic ranges. Overall, our study provides insight into the relative frequency of signatures of balancing and diversifying selection across the genome, but also identifies candidate genes that could be targeted with whole gene sequencing to further investigate these alternative modes of selection.

\section{Methods}

Sampling of Arabidopsis lyrata subspecies

Arabidopsis lyrata ssp. lyrata Seeds collected from individual plants were sampled at 12 sites (four selfing, and eight outcrossing populations) across the North American Great Lakes region in the summer of 2011 (Fig. 1a; described in detail in Buckley et al. 2016 [51]). A population previously described as mixed mating (TSSA) was grouped with the outcrossing samples because it shows similar patterns of diversity and heterozygosity to other outcrossing populations [51, 54]. In addition, seeds sampled in 2004 were included for one selfing population (TC), because no seeds were found in the 2011 field season. Seeds from six individual maternal plants from each population were germinated in a growth cabinet $\left(16: 8 \mathrm{~h}\right.$ day:night cycle; $20{ }^{\circ} \mathrm{C}: 16{ }^{\circ} \mathrm{C} ; 80 \%$ humidity), and DNA extracted from leaves from one individual from each of four separate maternal families per population. For TC, individuals germinated from only one maternal family, so in total there were $49 \mathrm{~A}$. l. lyrata individuals from different maternal families (Additional file 1: Table S1a).

Arabidopsis lyrata ssp. petraea Samples from European populations were collected from individual plants in the summer of 2007, apart from the leaf tissue from German and Austrian plants, which was collected in 2012 (Additional file 1: Table S1b). Although A. l. petraea is known to vary in ploidy across its range [49], only confirmed diploid populations were included [63]; Mable, unpublished). Seeds from the Scottish population were generously provided by Elizabeth Bourne. Seeds were germinated and grown in the same growth cabinet conditions as described for A. l. lyrata, and DNA extracted from leaves of one individual from 2 to 3 maternal families per population (Fig. 1b; Additional file 1: Table S1b). From central Europe, DNA was extracted from field-collected leaf tissue from 3 individuals from Germany and 2 from Austria. In total, 42 diploid individuals from A. l. petraea were used (Fig. 1b).

\section{DNA extraction, RAD library preparation and sequencing}

Fresh leaf tissue was dessicated using Drierite drying agent (WA Hammon Drierite Co. LTD, Xenia, US). DNA was extracted following the protocol of the Qiagen DNeasy plant mini kit (Qiagen, Manchester, UK), from dried leaf tissue, disrupted using a Fastprep machine and Fastprep lysing matrix A tubes (MP Biomedicals, Santa Ana, California, USA). DNA extractions from multiple leaves of an individual were pooled to obtain a minimum 
of $2 \mu \mathrm{g}$ per individual (at least $10 \mathrm{ng} / \mathrm{ul}$ ). DNA quantification and quality checks were performed using a Nanodrop ND1000 spectrophotometer and 2\% agarose gel electrophoresis. Final quantifications were performed using high-sensitivity double-stranded DNA assay kits for the Qubit 2.0 Fluorometer (Life technologies Ltd., Paisley, UK).

DNA from the 91 individuals was sent to Edinburgh Genomics (Edinburgh, UK) for 100 bp paired-end Restriction Associated DNA sequencing. DNA was digested using Pst 1 , which is not sensitive to methylation, and was predicted to produce 64,056 RAD loci across the $207 \mathrm{Mbp}$ A. lyrata genome. Each individual was tagged using barcodes differing by at least 2 bp. Three Pst 1 libraries for the 49 North American individually barcoded samples were optimized and sequenced using one lane of an Illumina HiSeq 2000 sequencer. The process was then repeated for the 42 European samples.

Raw 100 bp reads were demultiplexed using the individual barcodes. FastQC (Babraham informatics, Cambridge, UK) was used to check read quality and to count total reads per individual. Reads were filtered to remove those with uncalled bases and those of low quality using the flags $-\mathrm{c}$ and $-\mathrm{q}$ in the Stacks program process_radtags [58]. A window that is $15 \%$ of the size of the read length is moved across each read and removes the read if the average quality score within the window drops below a minimum phred score of 10 . The reads were trimmed to $92 \mathrm{bp}$ to remove the ID tags. Using fastQC to inspect read quality after the above-described read filtering revealed that average read quality score showed a clear peak at 38 in all samples, and that the median base quality rarely falls below a quality score of 32 (with the lower interquartile values rarely dropping below 30 ). Therefore, the reads were of high quality for use in further analyses.

\section{Alignment of Illumina reads to reference genome and RAD locus assembly}

Reads were aligned to the A. lyrata ssp. lyrata reference genome [45] (Phytozome version 1.0) using bowtie version 0.12 .9 [77]. Specifically, we aligned reads with only one genome location ( $-\mathrm{m} 1$ in bowtie) to the eight chromosomes of A. lyrata, 197Mbp in length, using default parameter conditions (seed length of 28, maximum of two mismatches allowed within seed). The reference-aligned reads were then processed by Stacks $v 1.32[58,78]$ in order to assemble RAD loci for each individual by genomic position, and then call genotypes using a maximum likelihood framework [7, 78]. We also used the error correcting module rxstacks to reduce the potential for sequencing errors to inflate diversity estimates. Specifically, this module removed a small number of assembled RAD loci (on average 167 per sample) with highly negative log-likelihoods that likely represent sequencing errors and removes erroneous SNP calls (affecting only $0.01 \%$ loci on average; see Additional file 1 : methods for details). To strike a balance between accurately calling heterozygotes and retaining large numbers of reads (and loci), we adopted a minimum threshold of 10 reads per individual per locus (Additional file 1: Figure S12). The proportion of heterozygous nucleotide sites was unaffected by increasing this threshold beyond 10 (Additional file 1: Figure S12b).

Of the 64,056 predicted loci, the majority (77.1\%) were recovered in the catalog of all loci in all individuals. However, many of these loci are not present in all individuals. Approximately 51.7\% (European samples) or 60.6\% (North American samples) loci were assembled per individual (out of the 64,056 predicted), reducing to 40.7 and $42.5 \%$ after removing those loci with fewer than 10 reads per locus. The majority of loci were removed at the populations stage, when filtering was applied to select only loci present in all or at least $50 \%$ individuals. Only $13.9 \%$ of all predicted loci were present in all samples, increasing to $33.8 \%$ of all predicted loci when up to $50 \%$ missing data were allowed within the sample sets.

\section{Genetic structure, diversity and heterozygosity across multiple spatial scales in A. lyrata}

Nucleotide diversity was estimated as the average number of nucleotide differences for all pairwise comparisons of sequences within a regional group, averaged across RAD loci and divided by the length of the RAD sequence (92 bp), equivalent to the nucleotide diversity estimate of Nei (1987) [79]. Expected heterozygosity (with alleles equivalent to $92 \mathrm{bp}$ RAD haplotypes) was also estimated [79]. Observed heterozygosity was estimated as the number of loci heterozygous for the $92 \mathrm{bp}$ RAD haplotype divided by the total number of loci observed in that individual.

We then used those loci present in all 91 individuals to determine the degree of genetic structuring within and between regions. RaxML v8 [80] was used to reconstruct a Maximum likelihood tree based on genome-wide SNPs and IUPAC codes were used to indicate heterozygous sites for each individual; invariant sites were not considered. A General Time Reversible (GTR) model with gamma distributed rate heterogeneity was used to model nucleotide substitutions. A bootstrap analysis (1000 pseudoreplications) was conducted to assess confidence in branching relationships.

A PCA analysis was also conducted to visualise genetic clustering across all the samples using polymorphic loci present in all individuals. We used RAD haplotypes (92 bp alleles) rather than SNPs for these analyses. RAD haplotypes identified by Stacks were converted to alleles 
in Genepop format using the haplotypes.tsv Stacks file and a custom R script by Mark Ravinet (https://github.com/markravinet/haplotype_to_genepop). Using the $\mathrm{R}$ packages "adegenet" [81, 82] and "ade4" [83] locus allele frequencies were centred around zero to ensure variances were comparable across loci. The first two principal components were plotted against each other for visualising clustering of samples. Separate PCAs were conducted to assess clustering among Northern European populations $(N=37$, using polymorphic loci present in all of those individuals), among populations within the three different Scandinavian countries (Norway, Sweden and Iceland), and across all North American Great Lakes samples $(N=49)$. For North American samples we also compared patterns of genetic structure inferred using RAD loci to genetic structure inferred using eight microsatellites (data from Foxe et al. 2010 [54]).

Finally, we used RAD loci present in all individuals to estimate IBD relationships for regions with sufficient sampling of populations, specifically all eight North American outcrossing populations and the five populations in each of Norway, Sweden and Iceland. Pairwise $\mathrm{F}_{\mathrm{ST}}$ (Weir \& Cockerham) values were estimated using the R program "diveRsity" [84], and geographic distances among population pairs was calculated using the earth.dist function in the "fossil" package [85]. Finally, multiple regression on matrixes (MRM function in package "ecodist"; [86] was used to test the significance of the IBD relationships. Isolation by distance relationships and plots were based on the standardised genetic distance estimate Fst/1-Fst.

\section{Detecting signatures of balancing selection at multiple spatial scales}

Defining population subsets at multiple spatial scales

We used several complementary approaches to examine genome-wide patterns of polymorphism and divergence and help detect signatures of balancing selection, focusing analyses on three different subsets of samples from the two subspecies. The following sets of samples were defined:

NA-S: We tested whether excesses of heterozygosity and diversity in genes under balancing selection can be detected against an overall low background level of polymorphism using individuals from five selfing populations of $A$. l. lyrata ( $N=17$; with only one individual for TC; Fig. 1a).

EU-N and NA-O: We then identified regions of elevated polymorphism that overlap with regions of reduced population structure or intermediate allele frequencies (elevated Tajima's D) using two sets of outcrossing populations sharing a recent demographic history and similar geographic coverage (area and distance among samples). For A. l. petraea we used samples from three Northern European countries (EU-N; 5 populations from each of Iceland, Norway and Sweden; $2-3$ individual per population, $N=35$; Fig. $1 \mathrm{~b}$ ) and for $A$. $l$. lyrata we used all North American Great Lakes outcrossing samples (NA-O; 8 populations, four individuals per population, $N=32$; Fig. 1a).

ALL-O: We then compared divergent lineages to increase our power to test for signatures of reduced population structure that overlap with genomic regions of elevated polymorphism. We compared all $A$. l. lyrata NA-O samples and all $A$. $l$. petraea samples, including the EU-N samples, but also two samples from Scotland and five samples from Central Europe (Fig. 1b), the latter thought to be the ancestral origin of diversity of the species [48].

\section{Filtering threshold decisions and population genetic diversity statistics}

For the population genomic analyses, we focused on loci with up to $50 \%$ missing data (present in a minimum of $50 \%$ of individuals in a sample group: NA-S, NA-O, EU-N or ALL-O). We also tested the consequence of this relaxed filtering $(<50 \%$ missing data vs $0 \%$ missing data) for the detection of candidate loci using the NA-S samples.

For estimating observed heterozygosity, nucleotide diversity and expected heterozygosity, we used variation in RAD haplotypes (linked set of variants within each 92 bp RAD locus) rather than considering individual SNPs at RAD loci. This strategy was used because highly polymorphic genes might be expected to have multiple SNPs within a 100 bp region, so allelic diversity may be particularly high and would be missed just by using a SNP-based approach. Both $\pi$ and $H_{e}$ are calculated by the program Stacks, whereas $H_{o}$ was estimated from Stacks output. Specifically, observed heterozygosity $\left(H_{o}\right)$ was estimated as the proportion of heterozygous individuals at a RAD locus out of the total number of individuals genotyped at that locus. Nucleotide diversity $(\pi)$ was estimated as the average number of nucleotide differences for all pairwise comparisons of sequences within the set of samples, divided by the length of the RAD sequence (92 bp), which is equivalent to the estimate of Nei (1987). Expected heterozygosity (gene diversity, $H_{e}$ ) was also estimated (Nei, 1987, p.180) for more direct comparison with $H_{o}$.

To detect genomic regions showing high diversity, we adopted a smoothing algorithm implemented in Stacks [7] to generate smoothed distributions of $92 \mathrm{bp}$ haplotype-level $\pi$ and $H_{e}$, as described in Hohenlohe et al. (2010). Each smoothed window is defined by the value $\sigma$, where a window $6 \sigma$ in length is centred on a 
polymorphic RAD locus. The width of the window was set for different sampling groups to encompass on average 30 RAD loci. The average diversity measure of RAD loci within this window is then weighted by distance to the RAD locus ( $3 \sigma$ in each direction), so RAD loci in the centre of the window more strongly influence overall window polymorphism. Since observed heterozygosity would not be as meaningful across genes (i.e. because it is a binary rather than a quantitative trait), only $\pi$ and $H_{e}$ were compared using the smoothing approach. Bootstrap resampling (with 1,000,000 pseudoreplicates) was used to test for windows showing significantly higher diversity than the genome-wide average (as described in [7]). Windows of significantly elevated diversity were identified using the relaxed threshold of $p<0.05$, as we were primarily interested in comparing overlapping windows among sample groups rather than robustly identifying all significantly elevated diversity regions per se. When adjacent windows of significantly elevated diversity overlapped (i.e. separated by less than the value of $\sigma$ ), they were considered as a single genomic region of significantly elevated diversity for annotation. The number and ID of genes in regions of elevated diversity were then compared among the different sample groups (NA-S, EU-N, NA-O) to look for shared candidate loci.

\section{Identifying candidate genes in smoothed candidate regions}

To test the relative ability of each of the above strategies to detect regions under balancing selection, we focused on a test panel of $A$. lyrata genes annotated as associated with disease resistance. This set included all annotated Nucleotide Binding Site-Leucine Rich Repeat (NLR) genes, plus some additional loci (Additional file 1: Table S5). We subsequently refer to these as A. lyrata NLR genes. All $A$. lyrata NLR genes within a genomic region of interest (showing either elevated diversity alone, or elevated diversity combined with reduced $\mathrm{F}_{\mathrm{ST}}$ or elevated Tajima's D) were extracted from a downloaded gff file (A. lyrata_107_v1.0_gene_details.gff from Phytozome v107).

We also looked for evidence of balancing selection (elevated diversity, intermediate allele frequencies, reduced genetic differentiation among populations and allele sharing among diverged lineages) within this set of NLR genes in Brassicaceae species. In total 17 of these genes represented clear a priori candidates for being under balancing selection [39-41, 57, 66, 69, 71, 75, 87-90], so these represent strong a priori candidates to be detected by our approach (summarised in Additional file 1: Table S6).

We used a randomisation test to test the prediction that the density of NLR loci is higher in windows of significantly elevated diversity than outside these windows. The randomisation test estimated whether the average number of NLR genes per smoothed diversity window $(+/-1 \times \sigma$ either side of each polymorphic locus) was significantly greater in windows of significantly elevated diversity compared to windows of non-significantly elevated diversity. Windows were labelled as "high diversity" or "not high diversity", then this label was randomly permuted (without replacement) and the difference in density of NLR genes between the two categories recalculated. This was repeated 1000 times to produce a null distribution of differences to which the observed difference is compared. The $p$-value indicated the proportion of simulated differences that were greater than the observed difference. For comparison to the NLR genes, we used a set of Leucine Rich ReceptorReceptor-like kinases (LRR-RLKs), which are not expected to be at higher frequency in regions of elevated diversity [35]. We used a list of 223 LRR-RLK genes present in A. thaliana [91] and then searched for A. lyrata genes annotated as these loci, resulting in 253 genes present in the A. lyrata genome assembly.

\section{Identifying loci showing elevated observed heterozygosity} and diversity in a low variation background (NA-S samples) Given the low overall levels of heterozygosity and diversity across the selfing (NA-S) samples, we looked for candidate genes in regions of significantly elevated diversity (both nucleotide diversity and expected heterozygosity), initially based on single outliers and then based on smoothing across multiple RAD loci. The single outlier approach involved identifying loci with the highest $1 \%$ values of $\pi, H_{e}$ and $H_{o}$ (described in the Additional file 1). Smoothed windows that showed either a smoothed estimate of expected heterozygosity or nucleotide diversity with significance $\mathrm{p}<0.05$ were considered as 'high diversity windows'. Each window was $900 \mathrm{Kbp}$ wide ( $\sigma$ value of $150 \mathrm{Kbp})$, as the low number of polymorphic loci across the selfing samples meant a large window size was required to encompass on average $20 \mathrm{RAD}$ loci (or on average 33 RAD loci when up to $50 \%$ missing data allowed). Neighbouring windows of significantly elevated diversity were then merged to form high diversity genomic regions and annotated NLR genes identified. For this set of samples, we also tested whether changing the amount of missing data $(0 \%$ or $50 \%)$ affected the number of candidate regions and the number and IDs of NLR loci detected.

\section{Identifying loci showing elevated diversity, reduced genetic differentiation and intermediate allele frequencies in outcrossing populations (EU-N, NA-O and ALL-O samples)}

For North American outcrossing and Northern European samples we focused on elevated $\pi$ and $H_{e}$ to identify high diversity genomic regions, but also used evidence for balancing selection based on reduced 
population differentiation $\left(\mathrm{F}_{\mathrm{ST}}\right)$ and an intermediate site frequency spectrum (Tajima's D). These analyses were based on RAD loci present in a minimum of $50 \%$ of individuals in a sample group $(<50 \%$ missing data). For the weighted smoothing analysis, window size was adjusted to contain about $30 \mathrm{RAD}$ loci, using a $\sigma$ value of $60 \mathrm{Kbp}$ for both EU-N and NA-O sample groups (total window size $=360 \mathrm{Kbp}$ ). Regions of significantly elevated diversity were identified in both groups separately and annotated NLR genes identified.

We then identified genomic regions of elevated diversity that also overlapped with windows of reduced population differentiation $\left(\mathrm{F}_{\mathrm{ST}}\right)$ or an intermediate site frequency spectrum (positive Tajima's D values) within each of the NA-O and EU-N sample sets. $\mathrm{F}_{\mathrm{ST}}$ was calculated in Stacks using RAD haplotypes and AMOVA-based $\mathrm{F}_{\mathrm{ST}}$ estimates [92], based on equations for allelic data described in [93]. $\mathrm{F}_{\mathrm{ST}}$ was estimated within smoothed windows of identical size to the smoothed diversity estimates. We calculated pairwise $\mathrm{F}_{\mathrm{ST}}$ among sample groups clearly separated in the RaXML tree, specifically among the three EU-N countries and among three clusters of the NA-O populations (three clusters of outcrossing populations: IND, SAK, SBD vs MAN, PIN, PCR vs TSS, TSSA; Fig. 1c). Windows with low $\mathrm{F}_{\mathrm{ST}}$ values (in the bottom $5 \%$ of the distribution of all windows) whose genomic position overlapped with regions of significantly elevated diversity were identified.

Tajima's D could not be calculated using Stacks and was therefore calculated using individual SNPs (from a VCF output file from Stacks) in a sliding window analysis using the R package "PopGenome" [94]. Windows were $120 \mathrm{Kbp}(+/-\sigma)$ wide and shifted by $12 \mathrm{Kbp}$ each time (the average approximate distance between RAD loci) and Tajima's D estimated using polymorphic sites in that window. From the distribution of Taijam's D values across all windows, we selected $5 \%$ of the windows with the highest Tajima's D values (right hand tail of each histogram; Additional file 1: Figure S9). We compared the position of windows with these highest 5\% of Tajima's D values with the position of regions of significantly elevated diversity and identified all $A$. lyrata NLR genes in these overlapping regions. As D can be inflated by hidden genetic structure, these analyses were performed separately for each EU-N country in turn (Norway, Sweden and Iceland) and all NA-O samples together. NA-O samples were pooled as population divergence within the Great Lakes region was comparable to that observed within each Scandinavian country (Fig. 1c). In contrast to Stacks, the windows are based on genomic position (not centred on a RAD locus) and there was no option to weight loci based on distance to the centre of the window.
Finally, we compared populations of the strongly diverged $A$. lyrata subspecies. Windows of significantly elevated diversity across all outcrossing (All-O) samples and $\mathrm{F}_{\mathrm{ST}}$ between subspecies were identified as described above, but using a reduced window size of $50 \mathrm{Kbp}$ due to the higher RAD marker density across this sample set. Overlap between elevated diversity and reduced $\mathrm{F}_{\mathrm{ST}}$ windows were then examined for the presence of $A$. lyrata NLR genes.

\section{Additional file}

Additional file 1: Contains supplementary methods and results, in addition to supplementary Tables S1-S7 and supplementary Figures S1S12. (DOCX $26587 \mathrm{~kb}$ )

\section{Acknowledgements}

We thank Edinburgh GenePool, particularly Karim Gharbi and Timothee Cezard for advice on designing the RAD-seq experiment and for training in bioinformatics. We thank Hans Recknagel for his assistance in setting up Stacks and providing help analysing the RAD-seq data. Seeds from Scotland were kindly provided by Elizabeth Bourne.

\section{Funding}

This work was supported by a Natural Environment Research Council (NERC) project grant to BKM and EH (NE/H021183/1 and NE/H020691/1).

\section{Availability of data and materials}

The raw, demultiplexed fastq files can be found on the NCBI SRA database with reference SRP148549. The IDs for the demultiplexed FASTQ files for individual biosamples are SAMN09230090-SAMN09230180.

\section{Authors' contributions}

JB and BKM designed the study, and analysed the data. MAK and PV provided leaf and seed samples used for RAD-sequencing. EBH provided details about specific R-genes. JB, EBH, PV, MAK and BKM wrote and revised the manuscript. All authors read and approved the final manuscript

Ethics approval and consent to participate

Not applicable.

\section{Competing interests}

The authors declare that they have no competing interests.

\section{Publisher's Note}

Springer Nature remains neutral with regard to jurisdictional claims in published maps and institutional affiliations.

\section{Author details}

${ }^{1}$ Institute of Biodiversity, Animal Health and Comparative Medicine, College of Medical, Veterinary and Life Sciences, University of Glasgow, Glasgow G12 $8 \mathrm{QQ}$, UK. ${ }^{2}$ Adaptation to a Changing Environment, Institute of Integrative Biology, ETH Zürich, CH-8092 Zürich, Switzerland. ${ }^{3}$ School of Life Sciences, Warwick Crop Centre, University of Warwick, Wellesbourne CV35 9EF, UK.

${ }^{4}$ Centre for Organismal Studies (COS) Heidelberg, Biodiversity and Plant Systematics, Heidelberg University, D69120 Heidelberg, Germany. ${ }^{5}$ Plant Ecology and Nature Conservation Group, Wageningen University, P.O.Box 47, 6700, AA, Wageningen, The Netherlands.

Received: 23 December 2017 Accepted: 18 May 2018

Published online: 27 June 2018

References

1. Davey JW, Hohenlohe PA, Etter PD, Boone JQ, Catchen JM, Blaxter ML. Genome-wide genetic marker discovery and genotyping using nextgeneration sequencing. Nat Rev Genet. 2011;12(7):499-510. 
2. Emerson KJ, Merz CR, Catchen J, Hohenlohe PA, Cresko WA, Bradshaw WE, Holzapfel CM: Resolving postglacial phylogeography using high-throughput sequencing. Proceedings of the National Academy of Sciences United States of America 2010, 107:16197-16200.

3. Friis G, Aleixandre P, Rodriguez-Estrella R, Navarro-Siguenza AG, Mila B. Rapid postglacial diversification and long-term stasis within the songbird genus Junco: phylogeographic and phylogenomic evidence. Mol Ecol. 2016; 25(24):6175-95.

4. Kamdem C, Fouet C, Gamez S, White BJ. Pollutants and insecticides drive local adaptation in African malaria mosquitoes. Mol Biol Evol. 2017;34(5): 1261-75.

5. Mattila TM, Tyrmi J, Pyhäjärvi T, Savolainen O. Genome-wide analysis of colonization history and concomitant selection in Arabidopsis lyrata. Mol Biol Evol. 2017;34(10):2665-2677.

6. Bruneaux M, Johnston SE, Herczeg G, Merila J, Primmer CR, Vasemagi A. Molecular evolutionary and population genomic analysis of the nine-spined stickleback using a modified restriction-site-associated DNA tag approach. Mol Ecol. 2013;22(3):565-82.

7. Hohenlohe PA, Bassham S, Etter PD, Stiffler N, Johnson EA, Cresko WA Population genomics of parallel adaptation in threespine stickleback using sequenced RAD tags. PLoS Genet. 2010;6(2):e1000862.

8. Pais AL, Whetten RW, Xiang QJ. Ecological genomics of local adaptation in Cornus florida L. by genotyping by sequencing. Ecol Evol. 2017;7(1):441-65.

9. Catchen JM, Hohenlohe PA, Bernatchez L, Funk WC, Andrews KR, Allendorf FW. Unbroken: RADseq remains a powerful tool for understanding the genetics of adaptation in natural populations. Mol Ecol Resour. 2017;17(3): 362-5.

10. Fijarczyk A, Babik W. Detecting balancing selection in genomes: limits and prospects. Mol Ecol. 2015;24(14):3529-45.

11. Haas RJ, Payseur BA. Fifteen years of genomewide scans for selection: trends, lessons and unaddressed genetic sources of complication. Mol Ecol. 2016;25(1):5-23.

12. Foll M, Gaggiotti O. A genome-scan method to identify selected loci appropriate for both dominant and codominant markers: a Bayesian perspective. Genetics. 2008;180(2):977-93.

13. Asthana S, Schmidt S, Sunyaev S. A limited role for balancing selection. Trends Genet. 2005;21:30-2.

14. Amambua-Ngwa A, Tetteh KKA, Manske M, Gomez-Escobar N, Stewart LB, Deerhake ME, Cheeseman $\mathrm{IH}$, Newbold Cl, Holder AA, Knuepfer E, et al. Population genomic scan for candidate signatures of balancing selection to guide antigen characterisation in malaria parasites. PLoS Genet. 2012;8(11): e1002992.

15. Thomas JC, Godfrey PA, Feldgarden M, Robinson DA. Candidate targets of balancing selection in the genome of Staphylococcus aureus. Mol Biol Evol. 2012;29(4):1175-86.

16. Novikova PY, Hohmann N, Nizhynska V, Tsuchimatsu T, Ali J, Muir G, Guggisberg A, Paape T, Schmid K, Fedorenko OM, et al. Sequencing of the genus Arabidopsis identifies a complex history of nonbifurcating speciation and abundant trans-specific polymorphism. Nat Genet. 2016; 48(9):1077-82

17. Croze M, Zivkovic D, Stephan W, Hutter S. Balancing selection on immunity genes: review of the current literature and new analysis in Drosophila melanogaster. Zoology. 2016;34(10):2665-2677.

18. Charlesworth D. Balancing seleciton and its effects on sequences in nearby genome regions. PLoS Genet. 2006;2(4):e64: 0379-0384

19. Hedrick PW. Pathogen resistance and genetic variation at $\mathrm{MHC}$ loci. Evolution; international journal of organic evolution. 2002;56:1902-8.

20. Delph LF, Kelly JK. On the importance of balancing selection in plants. New Phytol. 2014;201(1):45-56.

21. Hedrick PW. Genetic polymorphism in heterogeneous environments: the age of genomics. Annu Rev Ecol Evol Syst. 2006;37(1):67-93.

22. Charlesworth B, Nordborg M, Charlesworth D. The effects of local selection, balanced polymorphism and background selection on equilibrium patterns of genetic diversity in subdivided populations. Genet Res. 1997;70:155-74.

23. Schierup MH, Vekemans $X$, Charlesworth D. The effect of subdivision on variation at multi-allelic loci under balancing selection. Genet Res. 2000; 76(1):51-62

24. Beaumont MA, Balding DJ. Identifying adaptive genetic divergence among populations from genome scans. Mol Ecol. 2004;13(4):969-80.

25. Tajima F. Statistical method for testing the neutral mutation hypothesis. Genetics. 1989;123:585-95.
26. Croze M, Wollstein A, Bozicevic V, Zivkovic D, Stephan W, Hutter S. A genome-wide scan for genes under balancing selection in Drosophila melanogaster. BMC Evol Biol. 2017;17(1):15.

27. Luikart G, Allendorf FW, Cornuet J-M, Sherwin WB. Distortion of allele frequency distributions provides a test for recent populatino bottlenecks. Am Genet Soc. 1992;89:238-47.

28. Baird NA, Etter PD, Atwood TS, Currey MC, Shiver AL, Lewis ZA, Selker EU, Cresko WA, Johnson EA. Rapid SNP discovery and genetic mapping using sequenced RAD markers. PLoS One. 2008;3(10):e3376.

29. Sabeti PC, Varilly P, Fry B, Lohmueller J, Hostetter E, Cotsapas C, Xie X, Byrne EH, McCarroll SA, Gaudet R, et al. Genome-wide detection and characterization of positive selection in human populations. Nature. 2007; 449(7164):913-8.

30. Lowry DB, Hoban S, Kelley JL, Lotterhos KE, Reed LK, Antolin MF, Storfer A. Breaking RAD: an evaluation of the utility of restriction site-associated DNA sequencing for genome scans of adaptation. Mol Ecol Resour. 2017;17(2): 142-52.

31. Lighten J, van Oosterhout C, Bentzen P. Critical review of NGS analyses for de novo genotyping multigene families. Mol Ecol. 2014;23(16):3957-72.

32. Mable BK, Kilbride E, Viney ME, Tinsley RC. Copy number variation and genetic diversity of MHC class Ilb alleles in an alien population of Xenopus laevis. Immunogenetics. 2015;67(10):591-603.

33. Gouin A, Legeai F, Nouhaud P, Whibley A, Simon JC, Lemaitre C. Wholegenome re-sequencing of non-model organisms: lessons from unmapped reads. Heredity. 2015;114(5):494-501.

34. Kreitman M, Di Rienzo A. Balancing claims for balancing selection. Trends in genetics : TIG. 2004;20(7):300-4.

35. Sekhwal MK, Li P, Lam I, Wang X, Cloutier S, You FM. Disease resistance gene analogs (RGAs) in plants. Int J Mol Sci. 2015;16(8):19248-90.

36. Steuernagel B, Periyannan SK, Hernandez-Pinzon I, Witek K, Rouse MN, Yu G, Hatta A, Ayliffe M, Bariana H, Jones JD, et al. Rapid cloning of diseaseresistance genes in plants using mutagenesis and sequence capture. Nat Biotechnol. 2016;34(6):652-5.

37. Guo YL, Fitz J, Schneeberger K, Ossowski S, Cao J, Weigel D. Genome-wide comparison of nucleotide-binding site-leucine-rich repeat-encoding genes in Arabidopsis. Plant Physiol. 2011;157(2):757-69.

38. Clark RM, Schweikert G, Toomajian C, Ossowski S, Zeller G, Shinn P, Warthmann N, Hu TT, Fu G, Hinds DA, et al. Common sequence polymorphisms shaping genetic diversity in Arabidopsis thaliana. Science. 2007;317(5836):338-42.

39. Bergelson J, Kreitman M, Stahl EA, Tian D. Evolutionary dynamics of plant Rgenes. Science. 2001;292(5525):2281-5.

40. Bakker EG, Toomajian C, Kreitman M, Bergelson J. A genome-wide survey of $\mathrm{R}$ gene polymorphisms in Arabidopsis. Plant Cell. 2006;18(8): 1803-18.

41. Rose L, Bittner-Eddy PD, Langley $\mathrm{CH}$, Holub E, Michelmore RW, Beynon JL. The maintenance of extreme amino acid diversity at the disease resistance gene, RPP13, in Arabidopsis thaliana. Genetics. 2004;166:1517-27.

42. Ellis J, Dodds PN, Pryor T. Structure, function and evolution of plant disease resistance genes. Curr Opin Plant Biol. 2000;3:279-84

43. Koenig D, Weigel D. Beyond the Thale: comparative genomics and genetics of Arabidopsis relatives. Nat Rev Genet. 2015;16(5):285-98.

44. Weigel D, Nordborg M. Population genomics for understanding adaptation in wild plant species. Annu Rev Genet. 2015;49:315-38.

45. Hu TT, Pattyn P, Bakker EG, Cao J, Cheng JF, Clark RM, Fahlgren N, Fawcett JA, Grimwood J, Gundlach H, et al. The Arabidopsis lyrata genome sequence and the basis of rapid genome size change. Nat Genet. 2011;43(5):476-81.

46. Ross-Ibarra J, Wright SI, Foxe JP, Kawabe A, DeRose-Wilson L, Gos G, Charlesworth D, Gaut BS. Patterns of polymorphism and demographic history in natural populations of Arabidopsis lyrata. PLoS One. 2008;3(6)

47. Muller MH, Leppala J, Savolainen O. Genome-wide effects of postglacial colonization in Arabidopsis lyrata. Heredity. 2008;100(1):47-58.

48. Clauss MJ, Mitchell-Olds T. Population genetic structure of Arabidopsis lyrata in Europe. Mol Ecol. 2006;15(10):2753-66.

49. Koch MA, Matschinger M. Evolution and genetic differentiation among relatives of Arabidopsis thaliana. Proc Natl Acad Sci U S A. 2007:104(15): 6272-7.

50. Hohmann N, Koch MA. An Arabidopsis introgression zone studied at high spatio-temporal resolution: interglacial and multiple genetic contact exemplified using whole nuclear and plastid genomes. BMC Genomics. 2017;18(1):810. 
51. Buckley J, Kilbride E, Cevik V, Vicente JG, Holub EB, Mable BK. R-gene variation across Arabidopsis lyrata subspecies: effects of population structure, selection and mating system. BMC Evol Biol. 2016;16:93.

52. Mable BK, Robertson AV, Dart S, Di Berardo C, Witham L. Breakdown of selfincompatibility in the perennial Arabidopsis lyrata (Brassicaceae) and its genetic consequences. Evolution; international journal of organic evolution. 2005;59(7):1437-48.

53. Mable BK, Adam A. Patterns of genetic diversity in outcrossing and selfing populations of Arabidopsis lyrata. Mol Ecol. 2007;16(17):3565-80.

54. Foxe JP, Stift M, Tedder A, Haudry A, Wright SI, Mable BK. Reconstructing origins of loss of self-incompatibility and selfing in north American Arabidopsis lyrata: a population genetic context. Evolution; international journal of organic evolution. 2010;64(12):3495-510.

55. Willi $Y$, Maattanen $K$. The relative importance of factors determining genetic drift: mating system, spatial genetic structure, habitat and census size in Arabidopsis lyrata. The New phytologist. 2011;189(4):1200-9.

56. Mable BK, Hagmann J, Kim ST, Adam A, Kilbride E, Weigel D, Stift M. What causes mating system shifts in plants? Arabidopsis lyrata as a case study. Heredity. 2017;118(1):52-63.

57. Gos G, Slotte T, Wright SI. Signatures of balancing selection are maintained at disease resistance loci following mating system evolution and a population bottleneck in the genus Capsella. BMC Evol Biol. 2012;12:152.

58. Catchen J, Amores A, Hohenlohe PA, Cresko WA, Postlethwait JH. Stacks: building and genotyping loci de novo from short-read sequences. Genes Genomes Genet. 2011;1:171-82

59. Bent AF, Kunkel BN, Brown KL, Schmidt R, Giraudat J, Leung J, Staskawicz B. RPS2 of Arabidopsis thaliana: a leucine-rich repeat class of plant disease resistance genes. Science. 1994;265:1856-60.

60. Borhan MH, Gunn N, Cooper A, Sigrun G, Tör M, Rimmer SR, Holub EB. WRR4 encodes a TIR-NB-LRR protein that confers broad-spectrum white rust resistance in Arabidopsis thaliana to four physiological races of Albugo candida. Mol Plant-Microbe Interact. 2008;21:757-68.

61. Schmickl R, Paule J, Klein J, Marhold K, Koch MA. The evolutionary history of the Arabidopsis arenosa complex: diverse tetraploids mask the western Carpathian center of species and genetic diversity. PLoS One. 2012;7(8):e42691.

62. Hohmann N, Schmickl R, Chiang T-Y, Lučanová M, Kolár F, Marhold K, Koch MA. Taming the wild: resolving the gene pools of non-model Arabidopsis lineages. BMC Evol Biol. 2014;14:224.

63. Schmickl R, Jørgensen MH, Brysting AK, Koch MA. Phylogeographic implications for the north American boreal-arctic Arabidopsis lyrata complex. Plant Ecolog Divers. 2008;1 (2):245-54.

64. Shafer ABA, Peart CR, Tusso S, Maayan I, Brelsford A, Wheat CW, Wolf JBW, Gilbert M. Bioinformatic processing of RAD-seq data dramatically impacts downstream population genetic inference. Methods Ecol Evol. 2016;

65. Holub EB. The arms race is ancient history in Arabidopsis, the wildflower. Nat Rev Genet. 2001;2:516-27.

66. Cork JM, Purugganan MD. High-diversity genes in the Arabidopsis genome. Genetics. 2005;170(4):1897-911.

67. Holub EB. Natural variation in innate immunity of a pioneer species. Curr Opin Plant Biol. 2007;10(4):415-24.

68. Staal J, Kaliff M, Bohman S, Dixelius C. Transgressive segregation reveals two Arabidopsis TIR-NB-LRR resistance genes effective against Leptosphaeria maculans, causal agent of blackleg disease. The Plant journal : for cell and molecular biology. 2006;46(2):218-30.

69. Stahl EA, Dwyer G, Mauricio R, Kreitman M, Bergelson J. Dynamics of disease resistance polymorphism at RPM1 locus of Arabidopsis. Nature. 1999;400:667-71.

70. Grant M, Godiard L, Straube E, Ashfield T, Lewald J, Sattler A, Innes RW, Dangl JL. Structure of the Arabidopsis RPM1 gene enabling dual specificity disease resistance. Science. 1995;269:843-6.

71. Ding J, Zhang W, Jing Z, Chen JQ, Tian D. Unique pattern of R-gene variation within populations in Arabidopsis. Mol Gen Genomics. 2007;277(6):619-29.

72. Spurgin LG, Richardson DS. How pathogens drive genetic diversity: MHC, mechanisms and misunderstandings. Proceedings Biological sciences / The Royal Society. 2010;277(1684):979-88.

73. Li J, Shen H, Wang H, Zhao M, Luo Z, Wu H. Diversifying selection is the dominant factor affecting the geographical variation of MHC class II genes in the Omei tree frog. J Zool. 2016;300(3):197-204.

74. Sperschneider J, Ying H, Dodds PN, Gardiner DM, Upadhyaya NM, Singh KB, Manners JM, Taylor JM. Diversifying selection in the wheat stem rust fungus acts predominantly on pathogen-associated gene families and reveals candidate effectors. Front Plant Sci. 2014;5:372.
75. Tian D, Araki H, Stahl E, Bergelson J, Kreitman M. Signature of balancing selection in Arabidopsis. Proc Natl Acad Sci U S A. 2002;99(17):11525-30.

76. McDowell JM, Dhandaydham M, Long TA, Aarts MGM, Goff S, Holub E, Dangl JL. Intragenic recombination and diversifying selection contribute to the evolution of downy mildew resistance at the RPP8 locus of Arabidopsis. Plant Cell. 1998;10:1861-74.

77. Langmead B, Trapnell C, Pop M, Salzberg SL. Ultrafast and memory-efficient alignment of short DNA sequences to the human genome. Genome Biol. 2009;10(3):R25.

78. Catchen J, Hohenlohe PA, Bassham S, Amores A, Cresko WA. Stacks: an analysis tool set for population genomics. Mol Ecol. 2013;22(11):3124-40.

79. Nei M. Molecular evolutionary genetics. New York: Columbia University Press; 1987.

80. Stamatakis A. RAxML version 8: a tool for phylogenetic analysis and postanalysis of large phylogenies. Bioinformatics. 2014;30(9):1312-3.

81. Jombart T. Adegenet: a R package for the multivariate analysis of genetic markers. Bioinformatics. 2008;24:1403-5.

82. Jombart T, Ahmed I. Adegenet 1.3-1: new tools for the analysis of genomewide SNP data. Bioinformatics. 2011:27:3070-1.

83. Dray $\mathrm{S}$, Dufour $A B$. The ade4 package: implementing the duality diagram for ecologists. J Stat Softw. 2007;22:1-20.

84. Keenan K, McGinnity P, Cross TF, Crozier WW, Prodöhl PA. diveRsity: an R package for the estimation of population genetics parameters and their associated errors. Methods Ecol Evol. 2013;4:782-8.

85. Vavrek MJ: fossil: palaeoecological and palaeogeographical analysis tools. Palaeontol Electron, 14:1T. http://palaeo-electronica.org/2011_1/238/index. html. 2011.

86. Goslee SC, Urban DL. The ecodist package for dissimilarity-based analysis of ecological data. J Stat Softw. 2007;22(7)

87. Sicard A, Kappel C, Josephs EB, Lee YW, Marona C, Stinchcombe JR, Wright SI, Lenhard M. Divergent sorting of a balanced ancestral polymorphism underlies the establishment of gene-flow barriers in Capsella. Nat Commun. 2015;6:7960.

88. Noël L, Moores TL, van der Biezen EA, Parniske M, Daniels MJ, Parker JE, Jones JDG. Pronounced intraspecific haplotype divergence at the RPP5 complex disease resistance locu in Arabidopsis. Plant Cell. 1999;11:2099-111.

89. Mauricio R, Stahl EA, Korves T, Tian D, Kreitman M, Bergelson J. Natural selection for polymorphism in the disease resistance gene Rps2 in Arabidopsi thaliana. Genetics. 2003;163:735-46.

90. Karasov TL, Kniskern JM, Gao L, DeYoung BJ, Ding J, Dubiella U, Lastra RO, Nallu S, Roux F, Innes RW, et al. The long-term maintenance of a resistance polymorphism through diffuse interactions. Nature. 2014;512(7515):436-40.

91. Gou X, He K, Yang H, Yuan T, Lin H, Clouse S, Li J. Genome-wide cloning and sequence analysis of leucine-rich receptor-like protein kinase genes in Arabidopsis thaliana. BMC Genomics. 2010;11:19.

92. Weir BS, Cockerham CC. Estimating F-statistics for analysis of population structure. Evolution; international journal of organic evolution. 1984;38:1358-70.

93. Bird CE, Karl SA, Smouse PE, Toonen RJ: Detecting and measuring genetic differentiation. In: Phylogeography and Population Genetics in Crustacea. Edited by Held C, Koenemann S, Schubart CD: CRC Press; 2011.

94. Pfeifer B, Wittelsbuerger U, Ramos-Onsins SE, Lercher MJ. PopGenome: an efficient Swiss army knife for population genomic analyses in R. Mol Biol Evol. 2014;31:1929-36.

95. Parker JE, Coleman MJ, Szabò V, Frost LN, Schmidt R, van der Biezen EA, Moores T, Dean C, Daniels MJ, Jones JDG. Arabidopsis downy mildew resistance gene RPP5 shares similarity to the toll and Interleukin-1 receptors with N and L6. Plant Cell. 1997;9:879-94. 